Check for updates

Cite this: RSC Adv., 2019, 9, 27911

\title{
An erythrocyte membrane coated mimetic nano- platform for chemo-phototherapy and multimodal imaging $\dagger$
}

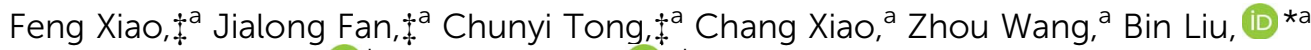 \\ Muhammad Daniyal (D) ${ }^{b}$ and Wei Wang (D)*b
}

\begin{abstract}
The tumor variability and low efficiency associated with conventional chemical drugs provide an impetus to develop drug-carrying systems with targeted accumulation and controllable release behavior. Herein, DOX-loaded Prussian blue (PB) nano-composites are developed after coating with erythrocyte membrane (EM) and modifying with folic acid (FA). In these nano-composites, PB nanoparticles mixture with different shapes were adopted to improve the photothermal performance, which is highly helpful for cancer photothermal ablation and controllable drug release. In addition, the nano-composites were endowed with high biocompatibility, immune evading capacity, $\mathrm{pH}$-/photo-responsive release behavior, and markedly prolonged blood circulation time, which was reflected by a $99.6 \%$ cervical tumor growth inhibition value (TGI) in vivo. Meanwhile, they functioned as multimodal bioimaging agents for photothermal, fluorescence, and photoacoustic imaging of tumors. The reported strategy can be applied for personalized therapy of various tumors by modifying the tumor-targeting molecule on the surface of nanoparticles.
\end{abstract}

Received 29th July 2019

Accepted 29th August 2019

DOI: $10.1039 / \mathrm{c} 9 \mathrm{ra} 05867 \mathrm{~b}$

rsc.li/rsc-advances

\section{Introduction}

Cervical cancer is the most common cancer in women worldwide, and it remains the leading cause of cancer-related deaths. ${ }^{1}$ Until now, several kinds of strategies have been developed for cervical cancer therapy. Among them, surgery is the treatment of choice for early invasive cancer. In more advanced cases, the combination of radiation with chemotherapy is the current standard of care. In patients with disseminated disease, chemotherapy or radiation provides symptom palliation. However, the indiscriminate tendency to kill normal cells and the destruction of the systemic immune system by chemical and radiation therapy severely impact the patient's quality of life after several treatments. ${ }^{2}$ Recently combinational therapy strategies based on nanomaterials have attracted wide attention for tumor therapy, due to the advantages of reducing the occurrence of the multi-drug resistance of tumor and the effective ability to deal with the "heterogeneity"

${ }^{a}$ College of Biology, Hunan University, Changsha, 410082, China. E-mail: binliu2001@hotmail.com; Fax: +86-731-89720939; Tel: +86-731-89720939

${ }^{b}$ TCM and Ethnomedicine Innovation \& Development International Laboratory, Innovative Materia Medica Research Institute, School of Pharmacy, Hunan University of Chinese Medicine, Changsha, 410208, P. R. China. E-mail: wangwei402@hotmail.com

$\dagger$ Electronic supplementary information (ESI) available. See DOI: 10.1039/c9ra05867b

\$ These authors contributed to the work equally and should be regarded as co-first authors. of cancer cells, etc..$^{3-5}$ Among of them, photothermal therapy (PTT), which employs a photothermal agent and near-infrared laser (NIR) for combination therapy of nano-systems, has recently emerged as an alternative with high selectivity and extremely low trauma. ${ }^{6,7}$ In this strategy, indocyanine green (ICG) is the most commonly used agent for PTT. ${ }^{8}$ However, this agent suffers from self-aggregation in physiological fluids, nonspecific binding to serum proteins and rapid clearance from blood circulation. ${ }^{9}$ In order to circumvent these limitations, several groups have used carbon and metal-based nanomaterials with good photothermal conversion efficiency in the NIR region for PTT with satisfying results for tumor ablation. ${ }^{10-12}$ However, the practical application of these photothermal agents including GO and CuS is still limited due to their unpredictable metabolic pathways and potential longterm accumulation toxicity in vivo. ${ }^{13-15}$ As one kind of metalorganic frameworks nanomaterials, Prussian blue nanoparticles (PB NPs) have been permitted by the U.S. Food and Drug Administration (FDA) for treating solid tumors. ${ }^{16}$ This kind of nanomaterial showed not only unparalleled advantages in drug-loading efficiency but also indicated excellent photothermal conversion efficiency in the NIR region, as well. ${ }^{17,18}$ Meanwhile, it can act as an emergent photoacoustic divergence agent using its strong optical absorption capacity in the NIR area. For example, Gu and Ye used carbon dots/PB NPs as optical nanoprobes for cancer cell imaging and photothermal conversion nano-formers for tumor PTT. ${ }^{19}$ In addition, PB-polyL-lysine (PLL) nanocomplex has been used as a highly efficient 
photoacoustic contrast agent for stem cells labelling and tracking. ${ }^{20}$

However, these nano-drug delivery systems based on bare PB NPs showed the short systemic circulation time, which extremely limited the drug accumulation at tumor sites. ${ }^{21}$ Meanwhile, the weak evading ability from macrophage uptake and strong blood clearance preference also reduced the therapy efficiency. In order to overcome these barriers, constructing a bio-mimetic nano-drug delivery system by adopting natural cell membranes become a new choice for improving drug accumulation at tumor region, extending half-life circulation and reducing immunogenicity, which has been primarily confirmed by the series study. ${ }^{22-25}$ Undoubtedly, the coating of the membrane system on nanoparticles improved the therapy efficiency through enhanced permeability and retention (EPR) effect in some extent, the passive accumulation of chemical drugs without actively aiming towards the solid tumors often resulted in high cost and unsatisfied tumor therapy outcomes. ${ }^{26-28}$ Meanwhile, these drug delivery systems with a fast release and wild distribution still caused considerable adverse effects to normal tissues.

Folate receptor is a glycosyl phosphatidyl inositol (GRI)linked membrane protein, which often highly expressed in several kinds of solid tumor including cervical carcinoma, hepatocarcinoma and human oral epidermoid carcinoma cells, etc. ${ }^{29,30}$ By using the high affinity to folate receptors, it has been widely used on drug delivery system for tumor targeting therapy, and the result demonstrated that the tumor-specific targeting nanocarriers obtained high efficient tumor therapy by improving cell uptake and targeted drug release. ${ }^{31,32}$ In this study, by combining the characteristics of PB NPs with an ultralarge surface area and hollow cavities, FA with specific targeting capacity and red cell membrane with immune stealthy ability, we constructed a smart and dual-modal nano-drug delivery system for combinational therapy of cervical carcinoma after evaluating its biocompatibility, loading capacity, release performance and cell-killing ability in vivo. The protocol proposed in Scheme 1 showed the preparation of SCPB@DOX@EM@FA NPs and its application in vitro and in vivo.

\section{Experimental}

\section{Preparation of spheric \& cubic hollow mesopores Prussian blue (SCPB NPs)}

The method of preparing cubic HMPB NPs was prepared as previously reported with modification. ${ }^{5}$ Briefly, $\mathrm{K}_{3}\left[\mathrm{Fe} \cdot(\mathrm{CN})_{6}\right]$. $3 \mathrm{H}_{2} \mathrm{O}(264 \mathrm{mg})$ and PVP $(3 \mathrm{~g})$ were dissolved in the $\mathrm{HCl}$ solution $(0.01 \mathrm{M})$ and stirred for $1 \mathrm{~h}$. Then, the yellow mixture was heated in an oil bath at $80{ }^{\circ} \mathrm{C}$ for $20 \mathrm{~h}$. After through centrifugation (12 $000 \mathrm{rpm}$ ), washing with water and vacuum drying, cubic PB NPs were acquired. Then, the obtained PB NPs (20 mg) and PVP $(100 \mathrm{mg}$ ) were mixed in the $\mathrm{HCl}$ solution and placed in a reaction vessel at $80{ }^{\circ} \mathrm{C}$ for $6 \mathrm{~h}$. The cubic HMPB NPs were collected after washing with water. The spheric hollow mesopores Prussian blue nanoparticles was obtained by change the amount of $\mathrm{K}_{3}\left[\mathrm{Fe} \cdot(\mathrm{CN})_{6}\right] \cdot 3 \mathrm{H}_{2} \mathrm{O}$ to $66 \mathrm{mg}$ under similar conditions. Then, the mixed of cubic and spheric HMPB NPs with different mass ratios were stirred $(800 \mathrm{rpm})$ for $4 \mathrm{~h}$ so as to obtain the cubic and spheric hollow mesopores Prussian blue nanoparticles.

\section{Preparation of erythrocyte membrane}

Whole blood taken from mice or human was washed with PBS (pH 7.4) and centrifuged at $3000 \mathrm{rpm}$ for $5 \mathrm{~min}$ to remove the plasma. After hemolysis in $0.25 \times \mathrm{PBS}(\mathrm{pH} 7.4)$ for $1 \mathrm{~h}$ at $4{ }^{\circ} \mathrm{C}$, the hemolyzed blood is centrifuged at $12000 \mathrm{rpm}$ for $10 \mathrm{~min}$ and washed with PBS ( $\mathrm{pH}$ 7.4) until the supernatant became colorless. The re-dispersed EM in PBS ( $\mathrm{pH} 7.4$ ) was sonicated at $4{ }^{\circ} \mathrm{C}$ for $30 \mathrm{~min}$. The broken EM was sequentially passed through $0.45 \mu \mathrm{m}$ and $0.2 \mu \mathrm{m}$ filter to get EM with uniform particle size. The content of membrane protein in EM was measured using the BCA protein assay kit.

\section{Preparation of SCPB@DOX NPs and SCPB@DOX@EM NPs}

The mixture containing $1 \mathrm{~mL}$ of SCPB NPs $\left(1 \mathrm{mg} \mathrm{mL}^{-1}\right)$ and $1 \mathrm{~mL}$ of DOX (1 mg mL $\left.\mathrm{mas}^{-1}\right)$ stirred at $800 \mathrm{rpm}$ for $12 \mathrm{~h}$. Subsequently, the unloaded DOX was removed through dialyzing using dialysis bag (MWCO of $3 \mathrm{kDa}$ ) for $12 \mathrm{~h}$ to obtain SCPB@DOX NPs solution. SCPB@DOX@EM NPs solution was prepared by adding EM $\left(1 \mathrm{mg} \mathrm{mL} \mathrm{m}^{-1}, 1 \mathrm{~mL}\right)$ into the SCPB@DOX NPs aqueous and stirred at $800 \mathrm{rpm}$ at $4{ }^{\circ} \mathrm{C}$ overnight.

\section{Preparation of SCPB@DOX@EM@FA NPs}

$100 \mathrm{mg}$ of EDC and $50 \mathrm{mg}$ of NHS were sequentially added into SCPB@DOX@EM NPs $\left(0.5 \mathrm{mg} \mathrm{mL} \mathrm{m}^{-1}, 2 \mathrm{~mL}\right)$ solution and stirred at $4{ }^{\circ} \mathrm{C}$ for $4 \mathrm{~h}$. After that, $10 \mathrm{mg}$ DSPE-PEG-NH $\mathrm{NH}_{2}$ was added into the mixed solution and stirred at $4{ }^{\circ} \mathrm{C}$ for $6 \mathrm{~h}$. Then, $1.5 \mathrm{mg}$ of FA was added and stirred at $4{ }^{\circ} \mathrm{C}$ overnight. The obtained solution was dialyzed using a dialysis bag (MWCO of $10 \mathrm{kDa}$ ).

\section{Drug loading and release in vitro}

Various concentrations of DOX $(125,250,500,1000,2000,3000$ $\left.\mu \mathrm{g} \mathrm{mL}{ }^{-1}, 500 \mu \mathrm{L}\right)$ were loaded into SCPB NPs $\left(1 \mathrm{mg} \mathrm{mL}^{-1}, 1 \mathrm{~mL}\right)$ to synthesize SCPB@DOX NPs. The concentration of DOX in the dialysis bag was measured on the microplate reader. The DOX loading efficiency (LC) and encapsulation efficiency (EC) were calculated according to the following eqn (1) and (2).

$$
\begin{aligned}
& \mathrm{LC}(\%)=\frac{M_{\mathrm{t}}-M_{\mathrm{u}}}{M_{\mathrm{p}}} \times 100 \% \\
& \mathrm{EC}(\%)=\frac{M_{\mathrm{t}}-M_{\mathrm{u}}}{M_{\mathrm{t}}} \times 100 \%
\end{aligned}
$$

where $M_{\mathrm{t}}, M_{\mathrm{u}}$, and $M_{\mathrm{p}}$ are the mass of the added DOX, the unencapsulated DOX, and the mass of SCPB NPs, respectively.

To investigate the release of DOX at different $\mathrm{pH}$ conditions, samples were divided into 4 groups including (1) SCPB@DOX NPs (pH 5.0), (2) SCPB@DOX NPs (pH 7.4), (3) SCPB@DOX@EM@FA NPs (pH 5.0), (4) SCPB@DOX@EM@FA NPs, PBS buffer (10 mM, pH 7.4) and acetate buffer (10 mM, pH 5.0). As for the DOX release efficiency, $1 \mathrm{~mL}$ of SCPB@DOX NPs $(2 \mathrm{mg}$ $\mathrm{mL}^{-1}$ ) solution sealed in the dialysis bag were immersed in 


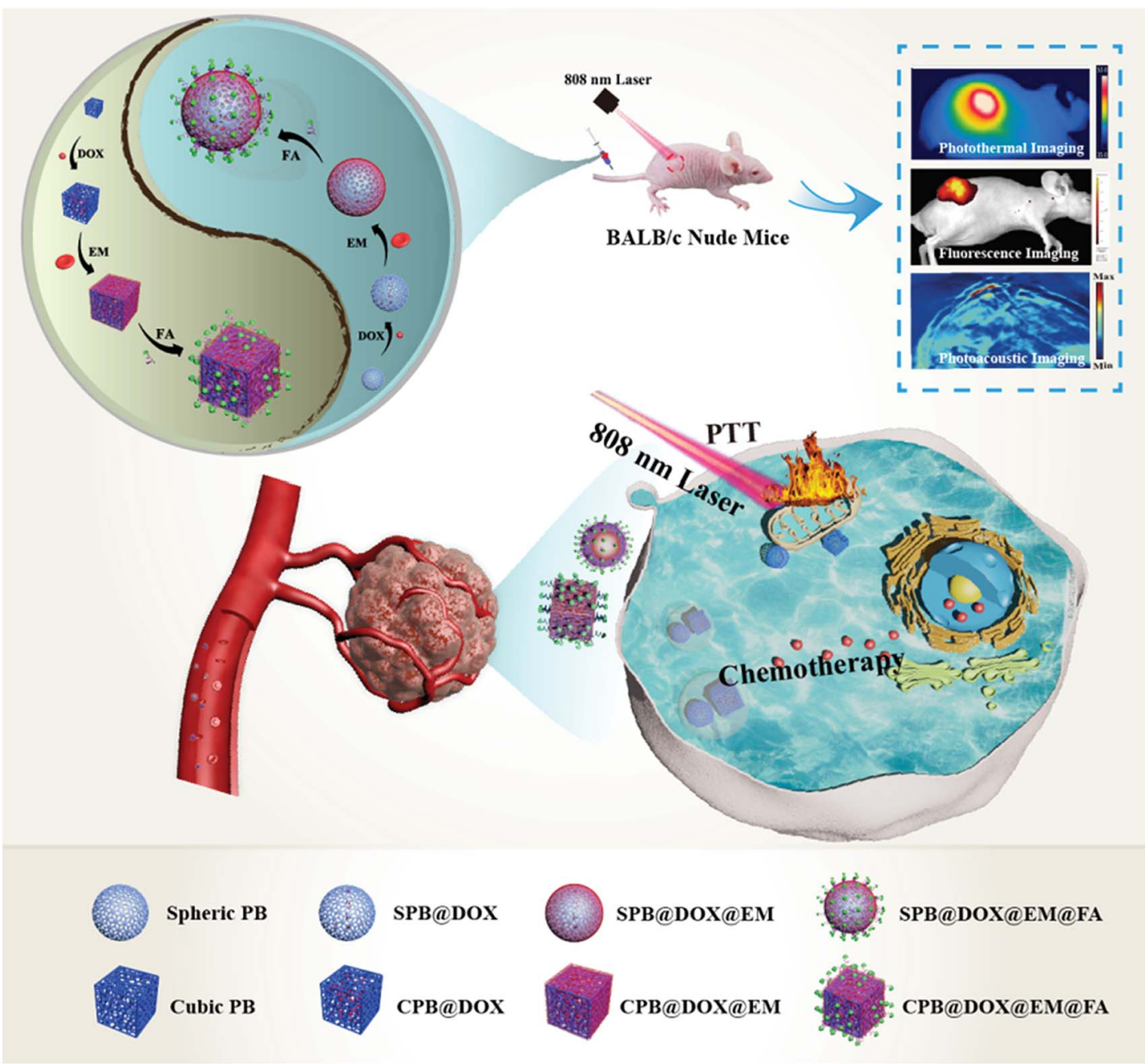

Scheme 1 Illustration of the preparation and anti-tumor application of drug loaded SCPB@DOX@EM@FA NPs.

$20 \mathrm{~mL}$ of PBS buffer ( $\mathrm{pH}$ 7.4) or $20 \mathrm{~mL}$ acetate buffer ( $\mathrm{pH} 5.0$ ). The same treatment was performed for SCPB@DOX@EM@FA NPs $\left(2 \mathrm{mg} \mathrm{mL}{ }^{-1}\right)$ solution. $2 \mathrm{~mL}$ of dialysate was taken out at intervals of $12 \mathrm{~h}$ and the same volume of the fresh PBS solution or acetate solution was replenished. The amount of released DOX was monitored using a microplate reader. The nearinfrared light-triggered release of DOX was studied by treating 2 mL of SCPB@DOX@EM@FA NPs solution with 4 cycles of the laser on/off under different $\mathrm{pH}$ values $(\mathrm{pH} 5.0$ or 7.4) using an $808 \mathrm{~nm}$ NIR laser $\left(0.8 \mathrm{~W} \mathrm{~cm}^{-2}, 10 \mathrm{~min}\right.$ for laser on, $20 \mathrm{~min}$ for laser off).

\section{Photothermal effect of SCPB@DOX@EM@FA NPs:}

EP tubes containing $0.5 \mathrm{~mL}$ of PBS (pH 7.4), SCPB@DOX NPs (0.2 mg mL $\left.\mathrm{mL}^{-1}\right)$, SCPB@DOX@EM NPs $\left(0.2 \mathrm{mg} \mathrm{mL} \mathrm{mL}^{-1}\right)$ or SCPB@DOX@EM@FA NPs $\left(0.2 \mathrm{mg} \mathrm{mL}{ }^{-1}\right)$ were irradiated with the near-infrared light $\left(808 \mathrm{~nm}, 0.8 \mathrm{~W} \mathrm{~cm}^{-2}\right.$ ) for $5 \mathrm{~min}$ and the temperature was recorded using a thermal infrared imaging camera every $1 \mathrm{~min}$. To investigate the photostability of SCPB@DOX@EM@FA NPs, 1 mL SCPB@EM@FA NPs (0.2 mg

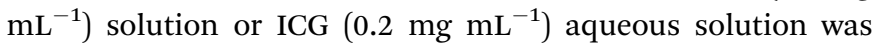

irradiated with $808 \mathrm{~nm}$ laser for $5 \mathrm{~min}$ and then cooled down to the room temperature in $5 \mathrm{~min}$. After repeating for 6 cycles, the UV-Vis absorbance spectra of the two solutions before and after the irradiation were recorded.

\section{Intracellular DOX release}

HeLa, MDA-MB-231, NIH-3T3, SMMC-7721 and BGC-823 cells were cultured in DMEM medium containing 10\% Fetal Bovine Serum (FBS) and 1\% streptomycin/penicillin, respectively. Cells were cultured at $37{ }^{\circ} \mathrm{C}$ in a humidified atmosphere with $5 \%$ $\mathrm{CO}_{2}$. Cell samples divided into 5 groups were subjected with different treatment: (1) DOX, (2) SCPB@DOX NPs, (3) SCPB@DOX@EM NPs, (4) SCPB@DOX@EM@FA NPs, (5) SCPB@DOX@EM@FA NPs, near-infrared (808 nm, $0.8 \mathrm{~W} \mathrm{~cm}^{-2}$ ) for $5 \mathrm{~min}$. HeLa cells were seeded into confocal dishes at a density of $5 \times 10^{4}$ cells per dish and incubated for $24 \mathrm{~h}$. Then, the medium was replaced by fresh medium containing DOX, SCPB@DOX NPs, SCPB@DOX@EM NPs, and SCPB@DOX@EM@FA NPs and incubated at $37^{\circ} \mathrm{C}$ for $3 \mathrm{~h}$. The concentration of DOX and SCPB were $10 \mu \mathrm{g} \mathrm{mL}{ }^{-1}$ and $20 \mu \mathrm{g} \mathrm{mL}{ }^{-1}$, respectively. After that, the cells of the group (5) was irradiated under 
NIR for $5 \mathrm{~min}$ and incubated at $37^{\circ} \mathrm{C}$ for $1 \mathrm{~h}$. Meanwhile, the cell nuclei were stained with Hoechst 33342 for $30 \mathrm{~min}$. The cells were observed under a laser scanning confocal microscope.

\section{Hemolysis assay}

A hemolysis assay was performed using fresh human blood samples provided by the People's Hospital of Hunan province. The erythrocytes were collected via centrifugation at $3000 \mathrm{rpm}$ for $5 \mathrm{~min}$ at $4{ }^{\circ} \mathrm{C}$, and washed 5 times with PBS solution to get pure erythrocytes. Afterward, $50 \mu \mathrm{L}$ of $4 \%$ erythrocytes (v/v) was mixed with $950 \mu \mathrm{L}$ of SCPB@DOX NPs, SCPB@DOX@EM NPs or SCPB@DOX@EM@FA NPs solution (dissolved in PBS with pH $7.4)$ at various concentrations $\left(25,50,100\right.$ and $\left.200 \mu \mathrm{g} \mathrm{mL}{ }^{-1}\right)$, and the resulting mixture was incubated at $37{ }^{\circ} \mathrm{C}$ for $4 \mathrm{~h}$. The positive control used erythrocytes mixed with pure water as $100 \%$ hemolysis. The samples were centrifuged at $3000 \mathrm{rpm}$ for 5 min at $4{ }^{\circ} \mathrm{C}$, and the absorbance of the supernatant at $540 \mathrm{~nm}$ was measured using a UV-Vis spectrophotometer. The percentage of hemolysis was calculated using the formula (3).

$$
\text { Hemolysis }(\%)=\left(I / I_{0}\right) \times 100 \%
$$

wherein $I$ represent the absorbance of the supernatant after addition of erythrocytes suspension by SCPB@DOX NPs, SCPB@DOX@EM NPs or SCPB@DOX@EM@FA NPs, and $I_{0}$ represents the absorbance of erythrocytes after complete hemolysis in pure water. All hemolysis experiments were performed in triplicate.

\section{MTT assay}

The cytotoxicity of SCPB@EM@FA NPs to HeLa, MDA-MB-231, NIH-3T3, SMMC-7721, and BGC-823 cells were evaluated. Briefly, cells seeded into 96-well plate were cultured for $24 \mathrm{~h}$. After washing with PBS, cells were exposed to the medium containing SCPB@EM@FA NPs (SCPB concentrations of 25, 50, 100 and $200 \mu \mathrm{g} \mathrm{mL}^{-1}$ ) for $24 \mathrm{~h}$. After washing with PBS for 3 times, $100 \mu \mathrm{L}$ of MTT solution $\left(0.5 \mathrm{mg} \mathrm{mL}^{-1}\right)$ was added and the cells were cultured for another $4 \mathrm{~h}$, the supernatant was carefully removed and $100 \mu \mathrm{L}$ of DMSO was added to each well. Cell viabilities were calculated according to the formula (4).

$$
\text { Cell viability }=\frac{\mathrm{OD}_{490 \mathrm{~nm} / \text { sample }}}{\mathrm{OD}_{490 \mathrm{~nm} / \mathrm{control}}} \times 100 \%
$$

After culturing for $24 \mathrm{~h}$, the medium of HeLa cells were replaced by fresh medium containing DOX $\left(1 \mu \mathrm{g} \mathrm{mL}^{-1}\right)$, SCPB NP $\left(20 \mu \mathrm{g} \mathrm{mL}^{-1}\right), \mathrm{SCPB} @ D O X$ NPs (SCPB concentration of $20 \mu \mathrm{g}$ $\mathrm{mL}^{-1}$, DOX concentration of $1 \mu \mathrm{g} \mathrm{mL}^{-1}$ ), SCPB@DOX@EM NPs (SCPB concentration of $20 \mu \mathrm{g} \mathrm{mL} \mathrm{m}^{-1}$, DOX concentration of $1 \mu \mathrm{g}$ $\mathrm{mL}^{-1}$ ), SCPB@DOX@EM@FA NPs (SCPB concentration of $20 \mu \mathrm{g}$ $\mathrm{mL}^{-1}$, DOX concentration of $1 \mu \mathrm{g} \mathrm{mL}^{-1}$ ) or PBS (10 mM, pH 7.4), respectively. $4 \mathrm{~h}$ later, the cells were irradiated with or without an $808 \mathrm{~nm}$ NIR laser $\left(0.8 \mathrm{~W} \mathrm{~cm}^{-2}\right)$ for $5 \mathrm{~min}$. The cells were further incubated for $20 \mathrm{~h}$ before the cell viability was assayed.

\section{Immune escape experiment}

All animal experiments were conducted in the Laboratory Animal Center of Xiangya School of Medicine under the guidance of the center. 5 female BALB/c mice ( 5 week old) were injected intraperitoneally with $1 \mathrm{~mL}$ of DMEM for 3 consecutive days. On the 4 th day, mice were intraperitoneally injected with $15 \mathrm{~mL}$ of DMEM. The abdomen of euthanized mice was licked for $20 \mathrm{~min}$. Then, the intra-abdominal tissue fluid was aspirated and centrifuged at $1000 \mathrm{rpm}$ for $5 \mathrm{~min}$. Obtained cells were cultured for $24 \mathrm{~h}$ after washing with the medium; then, the adherent macrophages were treated with lipopolysaccharide (LPS, the final concentration of $\left.1 \mu \mathrm{g} \mathrm{mL}^{-1}\right)$, SCPB@DOX NPs (0$\left.20 \mu \mathrm{g} \mathrm{mL}{ }^{-1}\right)$ or SCPB@DOX@EM@FA NPs $\left(0-20 \mu \mathrm{g} \mathrm{mL}{ }^{-1}\right)$ for another $12 \mathrm{~h}$ to collect the supernatant. The level of TNF- $\alpha$ was measured using an Elisa kit, and the adhered cells were imaged under a confocal microscope.

\section{In vitro specific targeting}

NIH-3T3, SMMC-7721, BGC-823, MDA-MB-231, and HeLa cells were seeded at a density of $1 \times 10^{5}$ cells per dish and incubated for $12 \mathrm{~h}$. To explore the targeting effect of FA modification, cells were pretreated with $10 \mu \mathrm{M}$ free FA for $30 \mathrm{~min}$. Then, the medium was replaced with fresh medium containing SCPB@DOX@EM@FA NPs (DOX concentration of $1 \mu \mathrm{g} \mathrm{mL}{ }^{-1}$ ). The cell nuclei were stained with Hoechst 33342 after culturing for $4 \mathrm{~h}$. All samples were imaged under a laser scanning confocal microscope.

\section{Apoptosis assay}

After culturing for $24 \mathrm{~h}$, HeLa cells were incubated with different materials containing PBS, SCPB NPs, DOX, SCPB@DOX NPs, SCPB@DOX@EM NPs or SCPB@DOX@EM@FA NPs (with DOX concentration of $0.5 \mu \mathrm{g} \mathrm{mL}{ }^{-1}$ ) for $4 \mathrm{~h}$, then, the cells were irradiated with or without an $808 \mathrm{~nm}$ NIR laser $\left(0.8 \mathrm{~W} \mathrm{~cm}^{-2}\right)$ for $5 \mathrm{~min}$ and were further incubated for $20 \mathrm{~h}$. The harvested cells were co-stained with Annexin V-Alexa Fluor 488 and PI detection kit for flow cytometry analysis.

\section{Calcein-AM/PI staining assays}

$5 \times 10^{5} \mathrm{HeLa}$ cells seeded into the 12 -well plate were cultured for $24 \mathrm{~h}$, followed by incubating with PBS, SCPB NPs, DOX, SCPB@DOX NPs, SCPB@DOX@EM NPs or SCPB@DOX@EM@FA NPs $\left(0.5 \mu \mathrm{g} \mathrm{mL}{ }^{-1}\right.$ DOX) for $4 \mathrm{~h}$. Then, cells were irradiated with or without an $808 \mathrm{~nm}$ NIR laser $\left(0.8 \mathrm{~W} \mathrm{~cm}^{-2}\right)$ for $5 \mathrm{~min}$. After incubating for another $20 \mathrm{~h}$, cells were stained with Calcein-AM/PI and imaged under the confocal microscope.

\section{Western blotting}

HeLa cells were incubated with cell culture medium containing PBS, SCPB NPs, DOX, SCPB@DOX NPs, SCPB@DOX@EM NPs or SCPB@DOX@EM@FA NPs $\left(0.5 \mu \mathrm{g} \mathrm{mL}^{-1} \mathrm{DOX}\right)$ for $4 \mathrm{~h}$. Then, the cells were irradiated with or without an $808 \mathrm{~nm}$ NIR laser $\left(0.8 \mathrm{~W} \mathrm{~cm}^{-2}\right)$ for $5 \mathrm{~min}$ and further incubated for $20 \mathrm{~h}$. The treated cells were lysed in the RIPA buffer. After separating with SDS-polyacrylamide gel electrophoresis (SDS-PAGE), proteins 
were transferred into nitrocellulose membranes. The membranes were incubated with antibodies against PARP, IKK, $\mathrm{NF}-\kappa \mathrm{B}$, caspase-3, BAX, Bcl-2 and $\beta$-actin overnight at $4{ }^{\circ} \mathrm{C}$ after blocking with $5 \%$ skim milk, followed by incubation with HRPconjugated secondary antibodies. The bands were visualized using the BIO-RAD ChemiDoc XRS chemiluminescence system.

\section{In vivo multimodal imaging}

Female BALB/c mice (5 week old) were used under regulation approved by the Laboratory Animal Center of the Xiangya School of Medicine, Central South University (Changsha, China). All the animal experiments were performed under the guidelines approved by the Medical Laboratory Animal Management Committee of the Xiangya School of Medicine. For in vivo imaging and investigating photothermal effects, $100 \mu \mathrm{L}$ of SCPB@ICG NPs, SCPB@ICG@EM NPs or SCPB@ICG@EM@FA NPs were injected intravenously into HeLa tumorbearing nude mice (with ICG concentration of $2.5 \mathrm{mg} \mathrm{kg}^{-1}$ ). ICG-labeling was used to avoid the interference of the fluorescence of the organism itself on imaging. In vivo fluorescent imaging was performed at $12,24,36$, and $48 \mathrm{~h}$ post-injection using a Kodak multimode imaging system $(\mathrm{Ex} / \mathrm{Em}=740 \mathrm{~nm} /$ $820 \mathrm{~nm})$. Mice $(n=3)$ were intravenously injected with $100 \mu \mathrm{L}$ of SCPB@DOX NPs, SCPB@DOX@EM NPs or SCPB@DOX@EM@FA NPs (2.5 mg kg ${ }^{-1}$ DOX) and then irradiated using NIR laser $\left(5 \mathrm{~min}, 808 \mathrm{~nm}\right.$, and $0.8 \mathrm{~W} \mathrm{~cm}^{-2}$ ) at $24 \mathrm{~h}$ post-injection, respectively. A thermal infrared camera was used to monitor the local temperature change of the tumor site. For in vitro distribution, the tumor and the major organs (heart, liver, spleen, lung, and kidney) were carefully collected at $48 \mathrm{~h}$ postinjection and imaged using Kodak multimode imaging system. After intravenous administration of $100 \mu \mathrm{L}$ SCPB@DOX@EM@FA NPs (SCPB concentration of $1 \mathrm{mg} \mathrm{mL}^{-1}$ ), the PA imaging of mice was acquired at $24 \mathrm{~h}$ post-injection using a commercial Endra Nexus 128 PA tomography system equipped with a tunable nanosecond pulsed laser.

\section{Pharmacokinetics and in vivo distribution}

The eyelid blood of tumor-bearing mice was collected at different time points $(0,1,2,4,8,12,24,48 \mathrm{~h})$ after vein administration with SCPB NPs, SCPB@DOX@EM@FA NPs (100 $\mu \mathrm{L}$, SCPB dosage at $5.0 \mathrm{mg} \mathrm{kg}^{-1}$ ). After injection for $24 \mathrm{~h}$, vital organs (heart, liver, spleen, lung, kidney) and tumors were collected and lysed in the mixture of $\mathrm{HClO}_{4}$ and $\mathrm{HNO}_{3}$ $\left(\mathrm{HClO}_{4}: \mathrm{HNO}_{3}=1: 10, \mathrm{v} / \mathrm{v}\right)$ for ICP-OES analysis.

\section{In vivo antitumor effect and safety evaluation}

To develop a tumor xenograft model, female BALB/c mice were subcutaneously injected with HeLa cells in the logarithmic growth phase. After reaching the tumor size of $100 \mathrm{~mm}^{3}$, mice were divided into 9 groups ( $n=3$ per group): (1) PBS, (2) PBS + laser, (3) DOX, (4) SCPB@DOX NPs, (5) SCPB@DOX NPs + laser, (6) SCPB@DOX@EM NPs, (7) SCPB@DOX@EM NPs + laser, (8) SCPB@DOX@EM@FA NPs, (9) SCPB@DOX@EM@FA NPs + laser. Nano-drug $\left(100 \mu \mathrm{L}, 2.5 \mathrm{mg} \mathrm{kg}^{-1}\right.$, DOX; $100 \mu \mathrm{L}, 5 \mathrm{mg} \mathrm{kg}^{-1}$, SCPB) was injected through the tail vein once every 2 days for 8 times in the first 16 days. After administration for $24 \mathrm{~h}$, the tumor site of the mouse was irradiated with $808 \mathrm{~nm}$ laser $(0.8 \mathrm{~W}$ $\left.\mathrm{cm}^{-2}\right)$ for $5 \mathrm{~min}$. Changes in tumor length $(L: \mathrm{mm})$ and width $(W: \mathrm{mm}$ ) were measured using vernier calipers. Body weight and tumor volume were recorded every other day. The tumor volume $(V)$ was calculated by the eqn (5):

$$
V=L \times W^{2} / 2
$$

After 18 days, vital organs (heart, liver, spleen, lung, and kidney), as well as tumors, were collected from euthanized mice and fixed with formalin. The paraffin sections of tumor and vital organs were performed for H\&E staining and TUNEL apoptosis assays. The tumor growth inhibition (TGI) rate was calculated according to eqn (6):

$$
\operatorname{TGI}(\%)=\frac{V_{\mathrm{c}}-V_{\mathrm{t}}}{V_{\mathrm{c}}} \times 100 \%
$$

where $V_{\mathrm{c}}$ represents the tumor volume of the control group, and $V_{\mathrm{t}}$ represents the tumor volume after the treatments. For biochemistry analysis blood plasma was obtained from mice blood after centrifugation.

\section{Statistical analysis}

SPSS version 22 was used for statistical analysis. All data were obtained in triplicate and presented as mean \pm standard deviation. One-way ANOVA was used followed by Tukey's post-test for all data analysis and $P$ value of ${ }^{*} P<0.05,{ }^{* *} P<0.01,{ }^{* *} P$ $<0.001$ was noted as statistically significant.

\section{Results and discussion}

\section{Characterization and photothermal properties of SCPB NPs}

First, various characterizing methods including XRD patterns, $\mathrm{N}_{2}$ adsorption-desorption isotherms, and the pore size distribution profile, FTIR spectra, UV-Vis spectra, were used to demonstrate the successful synthesis of the spheric, spheric \& cubic and cubic HMPB NPs, which was indicated as Fig. S1. $\dagger$ Meanwhile, SEM images confirmed the typical size of $130 \mathrm{~nm}$ and regular shape of HMPB NPs (Fig. 1A and B). Considering the light absorption property of PB NPs was reflected by the parameters of molar extinction coefficient $(\varepsilon)$ and the photothermal conversion efficiency $(\eta),{ }^{5,18}$ we then used eqn (S2) and $(\mathrm{S} 7) \dagger^{33,34}$ to determine their values and found that the cubic HMPB NPs possessing a larger nanoparticle volume in the same case showed a larger $\varepsilon$ value comparing with the spheric HMPB NPs $\left(1.17 \times 10^{13}\right.$ vs. $\left.2.8 \times 10^{13}\right)$, while the $\eta$ of the spheric HMPB NPs is larger than the cubic HMPB NPs $(47.75 \%$ vs. $37.05 \%)$, (see the ESI $\uparrow$ part 1 for the detailed calculations). As the heat energy release efficiency of NPs with near-infrared light exposure is closely related to the shape, size, etc. ${ }^{35-37}$ we proposed a hypothesis that the mixture of spheric and cubic HMPB NPs maybe improve the photothermal conversion performance, which is highly demanding for obtaining NIR-induced cancer photothermal ablation (PTA). In order to confirm it, the temperature of the samples containing spheric and cubic HMPB NPs mixtures (ratios from $4: 1$ to $1: 4$ ) under laser 
A

D
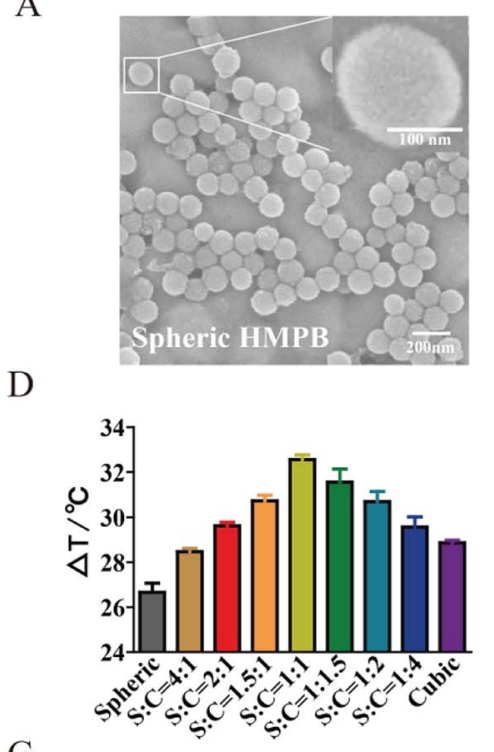

G

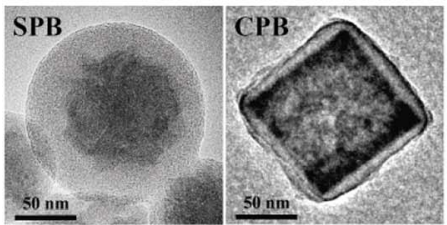

$\mathrm{J}$

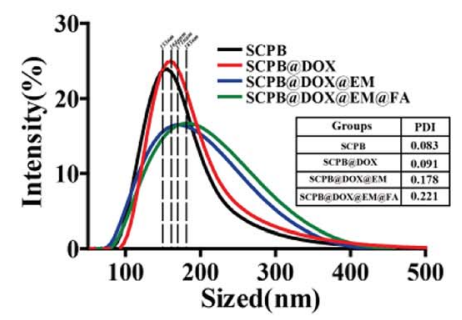

$\mathrm{H}$

B

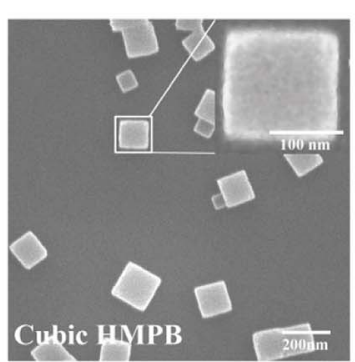

E
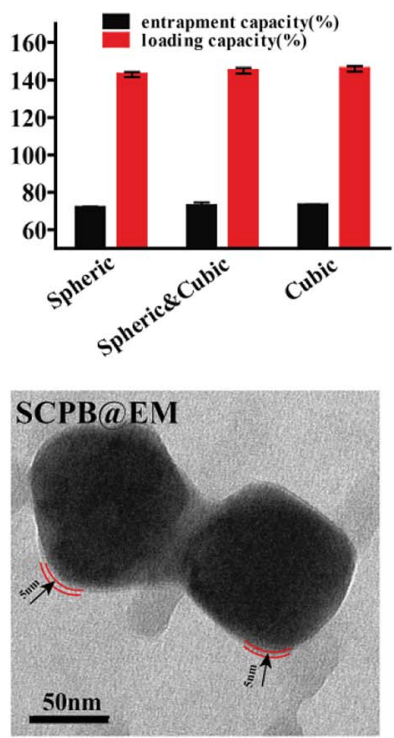

K

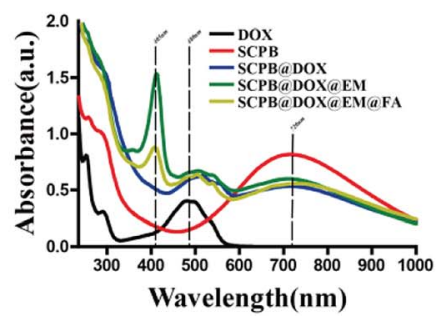

$\mathrm{C}$

Molar Extinction Coefficient $\left(\varepsilon, 10^{13}\right)$

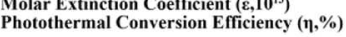

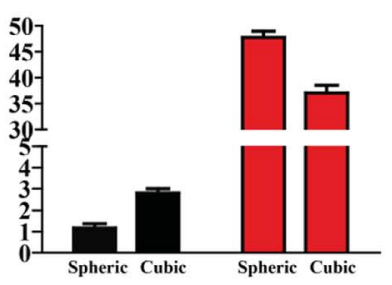

F
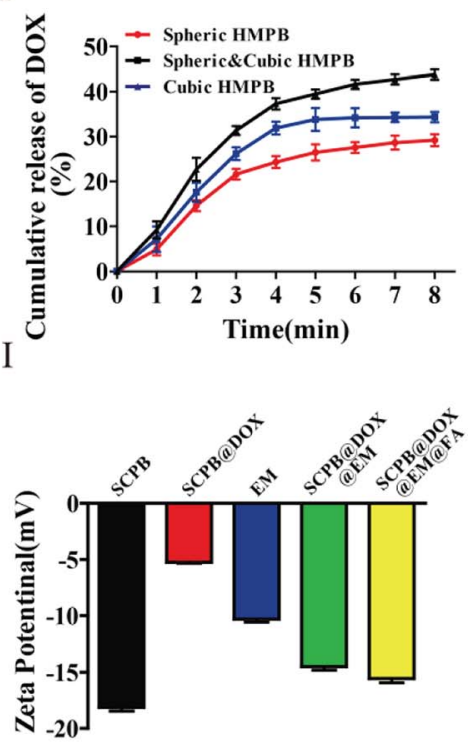

$\mathrm{L}$

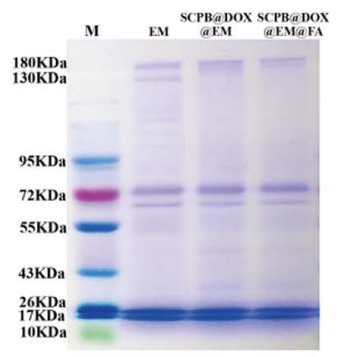

Fig. 1 (A and B) Scanning electron microscope (SEM) images. (C) The molar extinction coefficient and photothermal conversion efficiency of spheric, spheric \& cubic and cubic HMPB NPs. (D) Temperature change during $808 \mathrm{~nm}$ laser irradiation $\left(0.8 \mathrm{~W} \mathrm{~cm}{ }^{-2}\right)$ of the spheric and cubic with different ratios for $5 \mathrm{~min}$. (E) DOX loading efficiency and encapsulation efficiency of spheric, spheric $\&$ cubic and cubic HMPB NPs when the DOX concentration was $3.0 \mathrm{mg} \mathrm{mL}^{-1}$. (F) Controllable release of different DOX-loading NPs triggered by laser. (G) and (H) TEM of SPB NPs, CPB NPs and SCPB NPs with erythrocyte membrane modification (SCPB@EM NPs). (I) Surface charge of SCPB NPS, SCPBCDOX NPs, EM, SCPB@DOX@EM NPs, and SCPB@DOX@EM@FA NPs. (J) Size distribution and (K) UV-Vis absorption spectra of DOX, SCPB NPs, SCPB@DOX NPs, SCPB@DOX@EM NPs, and SCPB@DOX@EM@FA NPs. (L) SDS-PAGE of protein samples. Samples 1 to 4 is protein marker, sole erythrocyte membrane, SCPBCDOX@EM NPs, and SCPBCDOX@EMAFA NPs, respectively.

irradiation $\left(0.8 \mathrm{~W} \mathrm{~cm}^{-2}\right.$ for $\left.5 \mathrm{~min}\right)$ was investigated. The results in Fig. 1D showed that comparing to pure spheric and cubic HMPB NPs, the temperature increased in all mixture was maximum of $32.4{ }^{\circ} \mathrm{C}$ when the ratio is $1: 1$. This result indicates that the co-presence of two different kinds of HMPB NPs really improved the photothermal conversion performance, which is beneficial for triggering loaded drug release. As shown in Fig. 1E and $\mathrm{F}$, at the condition of same loading capacity and entrapment capacity, DOX release rate of mixture is higher than any pure NPs, which indicated the improved efficiency of DOX release under laser. Thus, the mixed PB NPs with improved photothermal conversion performance and drug release rate can be ideally used for combinational tumor theranostics.

\section{Physiochemical characterization of SCPB@DOX@EM@FA NPs}

Then, FA-modified erythrocyte membrane was used to coat DOX-loading SCPB to improve the biocompatibility and 
targeting the ability of this system. The TEM image in Fig. 1G and $\mathrm{H}$ showed the average size of SCPB NPs of $130 \mathrm{~nm}$, and a film coating of approximately $5 \mathrm{~nm}$ on the surface of SCPB NPs upon the erythrocyte membrane coating. In addition, the surface zeta potential of SCPB@DOX NPs changed from $-5.2 \mathrm{mV}$ to $-\mathbf{1 5 . 6} \mathrm{mV}$ following membrane coating and folic acid modification (Fig. 1I). Compared with DOX-loaded SCPB NPs (mean size of $164 \mathrm{~nm}$ ), the coating of the cell membrane and FA conjugation showed the weak increase in particle size and size distribution (171 nm and $185 \mathrm{~nm}$ ) (Fig. 1J). Meanwhile, the UV-Vis spectra of SCPB@DOX@EM@FA NPs showed characteristic absorption peaks including $714 \mathrm{~nm}$ of SCPB NPs, $480 \mathrm{~nm}$ of DOX and $405 \mathrm{~nm}$ of the erythrocyte membrane, respectively (Fig. 1K). Moreover, the confocal laser scanning microscope (CLSM) image directly indicated the successful encapsulation of SCPB NPs with erythrocyte membrane (Fig. S2A $\dagger$ ). In addition, the assay using SDS-PAGE method confirmed that nearly all of the endogenous membrane proteins were preserved on the SCPB@DOX@EM@FA NPs comparing with natural erythrocyte membrane (Fig. 1L). Furthermore, EDS analysis displayed the composition of SCPB@EM NPs with C, N, Fe and P elements, which also proved the camouflage of EM on the surface of SCPB NPs (Fig. S2B $\dagger$ ). FTIR spectroscopy (Fig. S2C $\dagger$ ) showed the absorbance peak at $2090 \mathrm{~cm}^{-1}$, which indicated $-\mathrm{CN}$ - stretching in the $\mathrm{Fe}-\mathrm{CN}-\mathrm{Fe}$ bond of SCPB NPs. The absorbance peak of SCPB@EM NPs at 1680 indicated the characteristic amide I band of the erythrocyte membrane (Fig. S2C $\dagger$ ). Comparing with pure SCPB NPs, there is no agglomeration, indicating the high stability of SCPB@DOX@EM@FA NPs. Together, these results indicate that spheric \& cubic HMPB NPs are successfully synthesized and the folate-targeted modified erythrocyte membrane effectively wraps the surface of SCPB NPs. Thus, the desired nanosystem was created.

\section{In vitro photothermal properties and drug release of SCPB@DOX@EM@FA NPs}

The charge transition between $\mathrm{Fe}^{2+}$ and $\mathrm{Fe}^{3+}$ ions induced by energy change under NIR light can result in a high molar extinction coefficient and photo-thermal property of PB NPs. ${ }^{38}$ By measuring the temperature change of different PB NPs under laser irradiation, it was found that the temperature of the PBS solution only increased $3.8^{\circ} \mathrm{C}$ after 5 min irradiation at a power density of $0.8 \mathrm{~W} \mathrm{~cm}{ }^{-2}$. However, the temperature of a solution containing SCPB NPs increased about $33.4{ }^{\circ} \mathrm{C}$ after with same treatment (from 30 to $63.4^{\circ} \mathrm{C}$ ) (Fig. 2A), which clearly reflected the efficient energy conversion of SCPB NPs induced by the laser irradiation. ${ }^{38}$ Moreover, the samples of SCPB@DOX NPs, SCPB@DOX@EM NPs, and SCPB@DOX@EM@FA NPs showed similar photothermal conversion efficiency comparing with SCPB NPs. These results indicate that the different modification including FA and EM negligibly affected the photothermal conversion efficiency of SCPB NPs, which is necessary for the following photothermal therapy. In addition, the photostability of SCPB@EM@FA NPs with ICG, an FDA-approved photothermal agent, was also compared. Fig. $2 \mathrm{~B}$ indicated that the temperature change caused by SCPB@EM@FA NPs is more than $30{ }^{\circ} \mathrm{C}$ after exposure to $808 \mathrm{~nm}$ for 6 heating-cooling cycle, which is significantly higher $\left(30^{\circ} \mathrm{C} v s .24^{\circ} \mathrm{C}\right)$ than that of ICG with the same treatment. These results clearly indicate its excellent photothermal stability SCPB@EM@FA NPs comparing with ICG. UV-Vis absorption spectra also confirmed it as no significant change of absorption peak was observed for SCPB@EM@FA NPs after with strong irradiation for $30 \mathrm{~min}$. In contrary, the peak of ICG almost disappeared (Fig. 2C). We then performed the drug loading assay under the different ratio of DOX/PB. As shown in Fig. 2D, the highest loading efficiency of SCPB NPs to DOX is $130.69 \%$ at the optimal ratio of DOX/PB with $3: 1$, which is comparable with previous reports. ${ }^{5}$ By combining with above result of pore-size distribution, nitrogen adsorption-desorption isotherm and UV-Vis absorption spectra, we suspected that the extremely high loading capacity can be attributed to the following factors: (1) the hollow mesoporous structure with a large cavity and numerous mesoporous (Fig. S1F and $\mathrm{G}^{\dagger}$ ); (2) the electrostatic interaction between positively charged DOX and negatively charged SCPB NPs (Fig. 2C), and the strong coordination bond between the $\mathrm{Fe}(\mathrm{III})$ inherent in the SCPB NPs structure and the amino or carbonyl group of DOX. ${ }^{18}$

As both high temperature and acidic conditions can directly destroy the erythrocyte membrane, which is beneficial for drug release, ${ }^{39}$ we first monitored the effect of $\mathrm{pH}$ on the DOX release behavior in the period of $72 \mathrm{~h}$ and found that the maximal drug cumulative rate of SCPB@DOX@EM@FA NPs at pH 5.0 increased about 2.5-fold compared with that of sample at $\mathrm{pH} 7.4$ (57.78\% vs. 22.2\%) (Fig. 2E). By further investigate the effect of laser irradiation on the DOX release under different $\mathrm{pH}$ conditions, it was found that the maximal drug cumulative rate at $\mathrm{pH}$ 5.0 increased to $79.70 \%$ after with 4 cycles of the laser on/off treatment. As a contrast, same treatment only resulted in the drug release to $66.04 \%$ at the condition of pH 7.4 (Fig. 2F). From the above data, it can be concluded that the release behavior of DOX is $\mathrm{pH}$ and temperature-dependent. More interestingly, the release rate of DOX is remarkably increased with the help of laser irradiation, which was reflected by the release curve slope change from 0.34 to 2.20 . These results mean that DOX can be released with precise dosage control under the stimulation of NIR laser during the whole treatment phase, which can significantly improve the utilization of chemical drugs.

\section{Biocompatibility and the immune evading ability of SCPB@DOX@EM@FA NPs}

Next, the biocompatibility of these nanomaterials from different aspects was evaluated. Fig. 3A indicated that all samples could not cause hemolysis (less than $3 \%$ hemolysis rate) after incubating with blood for $4 \mathrm{~h}$. Moreover, SCPB NPs with EM coating reduced hemolysis rate to $1 \%$ even at the 200 $\mu \mathrm{g} \mathrm{mL}^{-1}$. Meanwhile, these nanocomposites with the high concentration of $200 \mu \mathrm{g} \mathrm{mL} \mathrm{mL}^{-1}$ did not show obvious toxicity to cultured cells in vitro, which was reflected by the high cell viability (more than 85\%) of NIH-3T3, SMMC-7721, HeLa, MDAMB-231 and BGC-823 (Fig. 3B). In addition, the immune- 
A

C
(1)

(2)

(3)

(4)

(5)

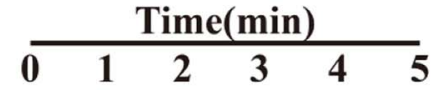
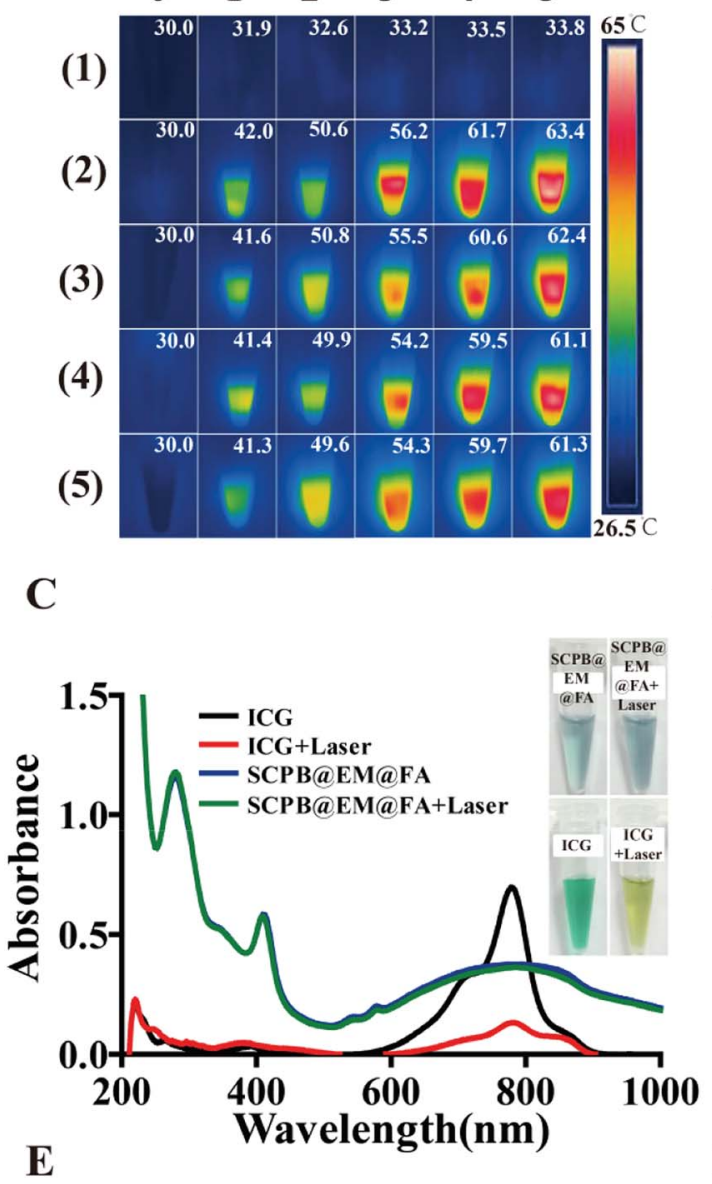

B

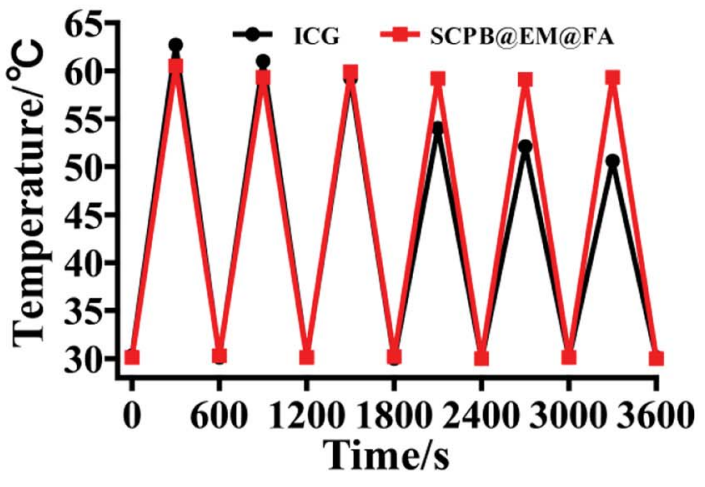

D

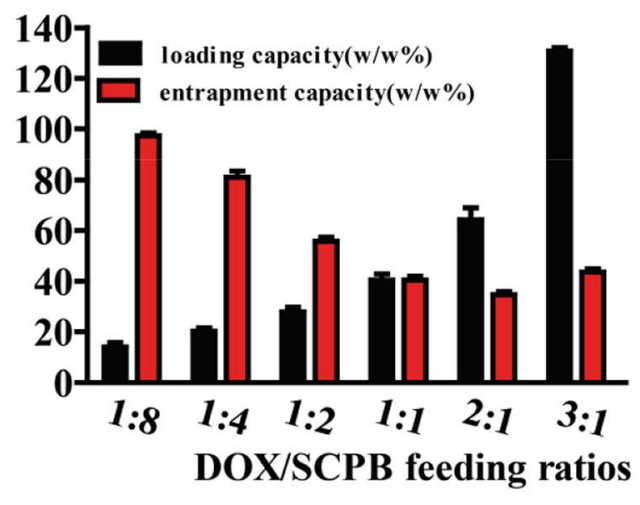

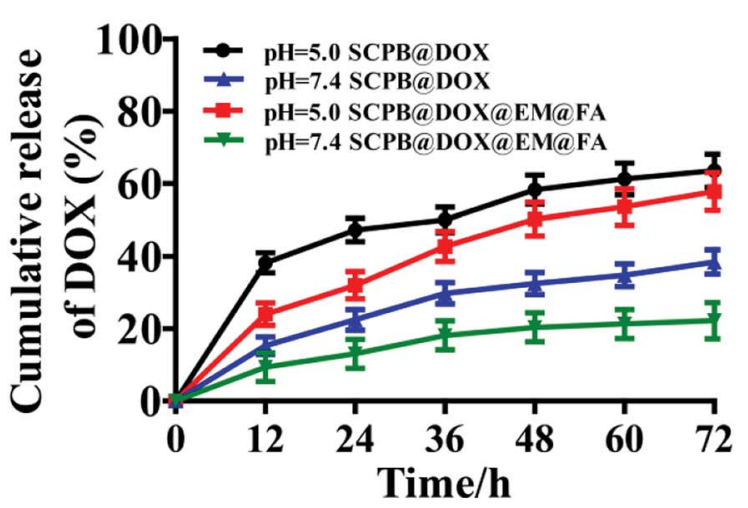

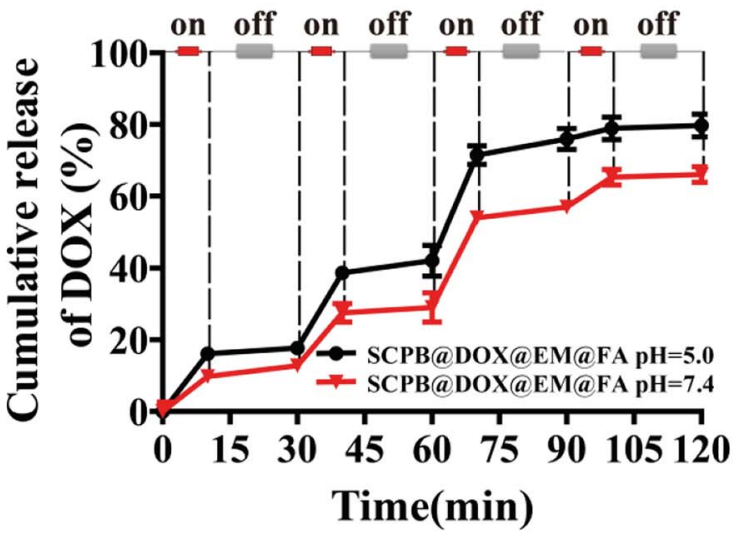

Fig. 2 (A) Infrared thermal images of (1) PBS, (2) SCPB NPs, (3) SCPB@DOX NPs, (4) SCPB@DOX@EM NPs and (5) SCPB@DOX@EM@FA NPs (with SCPB concentration at $0.2 \mathrm{mg} \mathrm{mL}^{-1}$ ) during $808 \mathrm{~nm}$ laser irradiation $\left(0.8 \mathrm{~W} \mathrm{~cm}^{-2}\right)$ for $5 \mathrm{~min}$. (B) Photostability of SCPB@EM(FA NPs (with SCPB concentration at $\left.0.2 \mathrm{mg} \mathrm{mL}^{-1}\right)$ under $808 \mathrm{~nm}$ laser irradiation $\left(0.8 \mathrm{~W} \mathrm{~cm}{ }^{-2}\right)$ using ICG as control and (C) UV-Vis spectra of ICG and SCPB@EM@FA NPs (with SCPB concentration at $0.2 \mathrm{mg} \mathrm{mL}^{-1}$ ) before and after $808 \mathrm{~nm}$ laser irradiation. Inset: images of SCPB@EM(AFA NPs and ICG before and after $808 \mathrm{~nm}$ laser irradiation. (D) DOX loading efficiency and encapsulation efficiency of SCPB@DOX NPS when the DOX concentration was $0.125,0.25,0.5,1.0,2.0$ and $3.0 \mathrm{mg} \mathrm{mL}^{-1}$, respectively. (E) DOX release from SCPB@DOX NPs and SCPB@DOX@EM@FA NPs at $\mathrm{pH} 5.0$ and 7.4. (F) DOX release behavior from SCPB@DOX@EM@FA NPs with 4 laser on/off cycles. All release studies were performed in triplicate.

evading assay indicated that macrophages emitted a strong red fluorescence signal with a concentration-depending manner after culturing with SCPB@DOX NPs. In contrary, the extremely weak red fluorescence signal was observed in macrophages incubating with SCPB@DOX@EM@FA NPs (Fig. 3C). These results demonstrate that the camouflage of EM significantly 
A

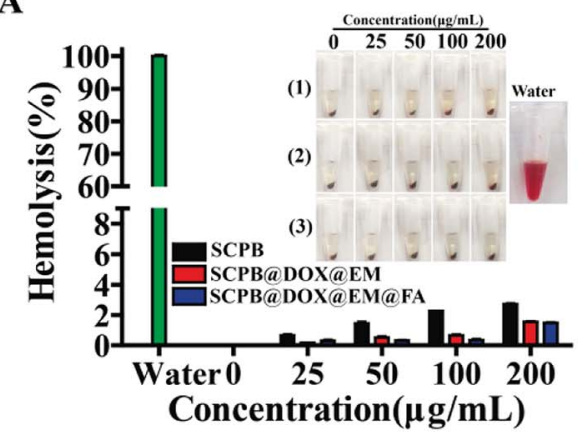

C

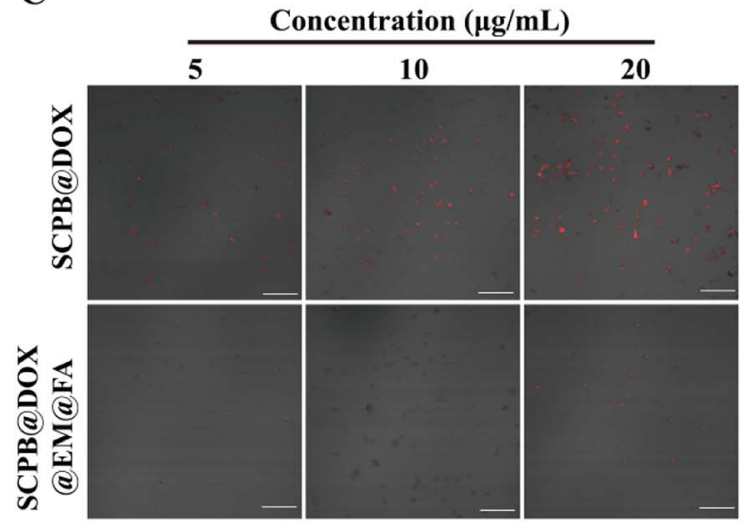

B

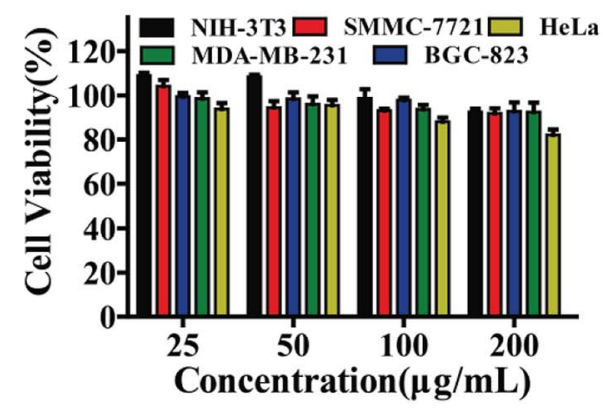

D

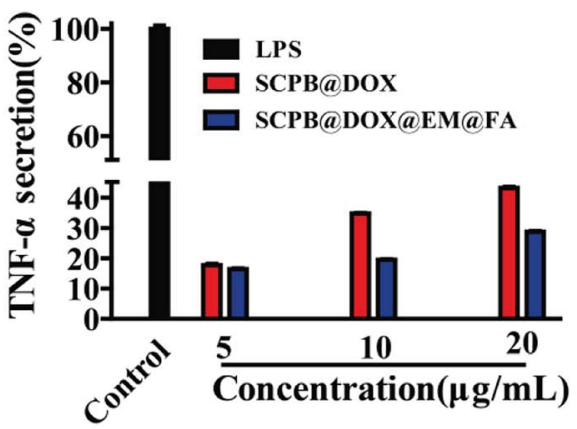

Fig. 3 (A) Hemolysis quantification of EM incubated with SCPB@DOX NPs, SCPB@DOX@EM NPs, and SCPB@DOX@EM@FA NPs. (B) Cytotoxicity of SCPB@EM@FA NPs to different cell lines. (C) The confocal scanning microscope images of macrophages after culturing with SCPB aDOX NPs or SCPBADOX@EMaFA NPs for $12 \mathrm{~h}$, the control was without treatment (scale bar $=50 \mu \mathrm{m}$ ). (D) The effect of SCPB@DOX NPs or SCPB@DOX@EM@FA NPs on the TNF- $\alpha$ secretion. The TNF- $\alpha$ levels in PBS and lipopolysaccharide (LPS)-treated macrophages were negative control (0\%) and positive control (100\%), respectively.

improved the immune stealthy ability of SCPB NPs from macrophages. ELISA assay also indicated that the TNF- $\alpha$ level in the SCPB@DOX@EM@FA NPs treatment group decreased by about 50\% comparing with SCPB NPs treatment group (Fig. 3D). In summary, EM-coated SCPB NPs efficiently reduced macrophage phagocytosis and immune response, which was beneficial for prolonging the blood circulation time and extending the half-life of SCPB@DOX@EM@FA NPs in vivo.

\section{Targeting and uptake mechanism of SCPB@DOX@EM@FA NPs}

As a tumor marker overexpressed in more than $90 \%$ of ovarian carcinomas, folate receptor (FR) has been widely used as an efficient molecular target for tumor therapy. ${ }^{40}$ We investigated the cytotoxicity and FR expression levels of FR-positive HeLa cell lines and FR-negative SMMC-7721 and normal NIH-3T3 cell lines. As shown in Fig. 4A, no difference was observed for the cell viability of SMMC-7721 and NIH-3T3 cells incubated with or without FR blockers, whereas the cell viability of FR-positive HeLa cells decreased significantly comparing with the FA block group. Meanwhile, the western blot also verified the high expression of FR in the HeLa cells compared with the other two cell lines. In addition, Fig. 4B indicated that red fluorescence signal in HeLa cells incubating with SCPB@DOX@EM@FA NPs is stronger than that of SCPB@DOX@EM NPs. Meanwhile, excessive FA treatment before adding SCPB@DOX@EM@FA NPs resulted in the significant fluorescence signal decrease of HeLa cells. These results suggest that SCPB@DOX@EM@FA NPs selectively accumulated in the HeLa cells with high FR expression.

Nanomaterials can pass through the cell membrane and enter into the lysosome, which is easily degraded by enzymes or acids and release into the cell cytoplasm. Therefore, the ability to enter into cells of nano-drug carriers directly determined the efficiency for tumor therapy. ${ }^{41}$ By investigating cell entry behavior of different nanomaterials system, we found that the cell uptake ability of SCPB@DOX NPs is more powerful than the naked DOX. Moreover, EM coating on the surface of SCPB@DOX NPs further enhanced the cellular uptake efficiency and lysosomal escape ability. Noteworthy, the amount of DOX in the nucleus of SCPB@DOX@EM@FA NPs increased sharply after with laser irradiation, which reflected the further improvement of lysosome escape of SCPB@DOX@EM@FA NPs and acceleration of drug release with the help of laser (Fig. 4C and S3†). The above results indicate that laser irradiation enhances the release of drug and the efficiency of entering the nucleus due to the excellent photothermal effect of SCPB NPs under laser irradiation.

The above findings of cell uptake motivated us to explore the hindered mechanism. After performing the inhibition experiment using three kinds of inhibitors ( $\beta$-cyclodextrin, rottlerin, and chloroquine), it was found that the presence of rottlerin, the 

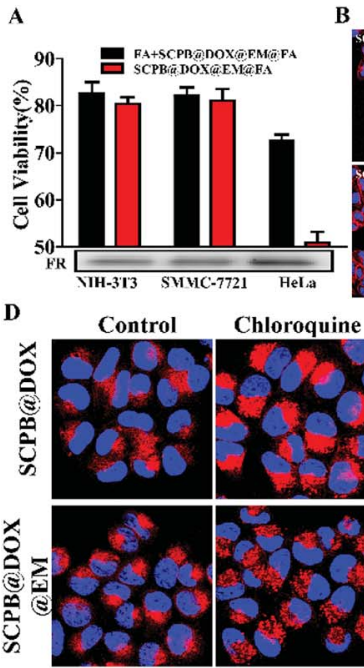
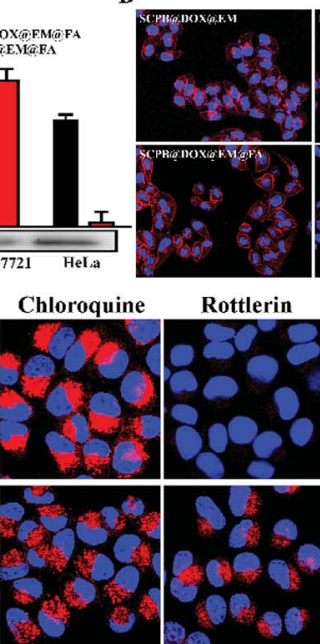

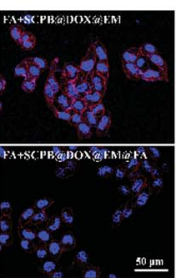

$\beta$-cyclodextrin

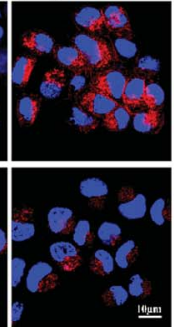

C
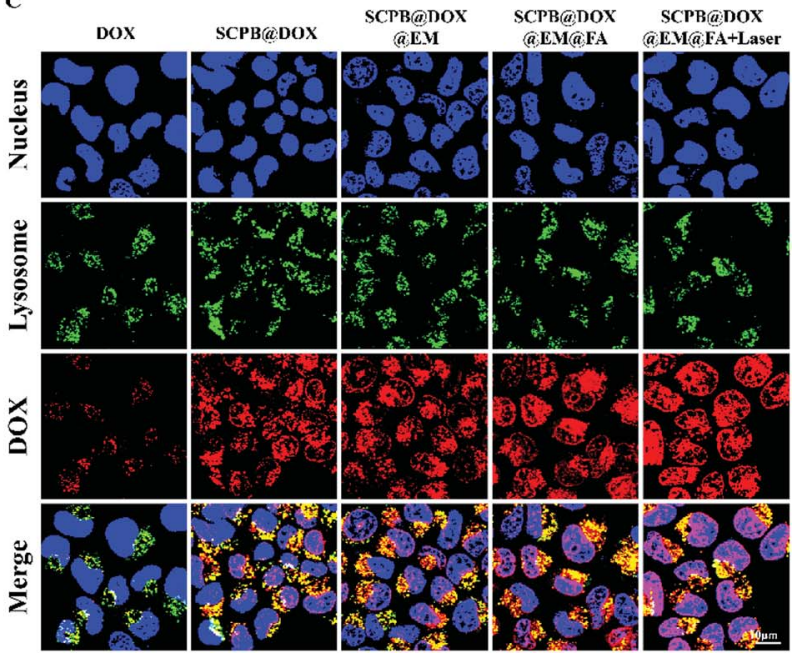

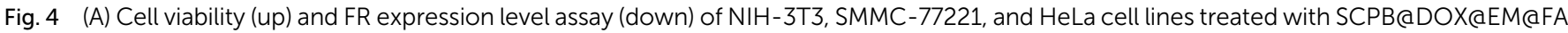

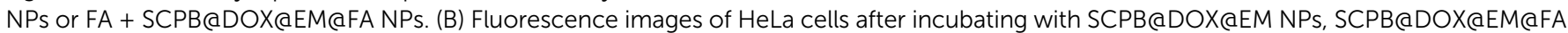

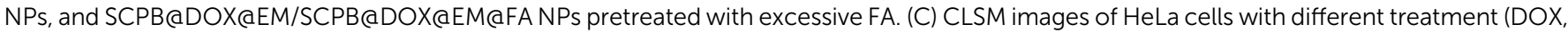

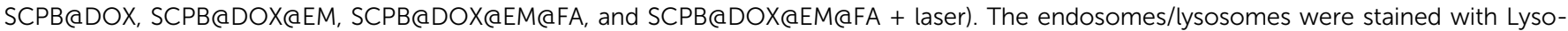

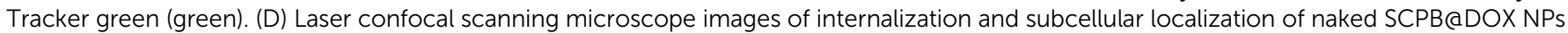
and erythrocyte membrane coated SCPBADOX NPs in the presence of three kinds of small-molecule inhibitors.

inhibitor of micropinocytosis, significantly reduced the uptake efficiency of tumor cells to the SCPB@DOX NPs, which was embodied by the red fluorescence intensity reduction in the rottlerin treatment group compared with the control. Quantitative assay indicated that the uptake efficiency of naked SCPB NPs reduced about $98 \%$ at the presence of rottlerin (Fig. 4D). These findings, which are consistent with the previous report, ${ }^{42}$ demonstrate that the anion nanomaterials like SCPB NPs enter into tumor cells mainly via micropinocytosis. Meanwhile, we found that the camouflage of SCPB NPs with EM significantly affected cell uptake manner. The significant reduction of uptake was observed for the presence of $\beta$-cyclodextrin, which can block caveolae-mediated endocytosis. Cellular uptake efficiency to SCPB@DOX@EM NPs reduced about 80\%. These results indicate that SCPB@DOX NPs enter cells through micropinocytosis by triggering actin-mediated membrane ruffling, while the SCPB@DOX@EM NPs enter into cells through caveolaemediated endocytosis. The mechanism of cell uptake difference with the two materials is investigating.

\section{In vitro anti-tumor activity}

The anti-cancer activity of SCPB@DOX@EM@FA NPs was assessed by Calcein-AM/propidium iodide staining in combination with MTT. Fig. 5A indicated that almost no apoptotic/dead cells were observed in the PBS or sole SCPB NPs treatment group without laser irradiation, indicating the favorable biocompatibility of this kind of nanocarrier. The dual treatment of SCPB@DOX with laser irradiation resulted in the increase of dead cell comparing with the free DOX treatment group. By contrast, almost all HeLa cells were killed after with combinational treatment of SCPB@DOX@EM@FA NPs and laser irradiation. Fig. 5B also indicated that neither laser irradiation nor SCPB NPs has significant cytotoxicity on HeLa cells. Cell death rate was about
60\% after with SCPB@DOX@EM@FA NPs treatment. However, the dual treatment of SCPB@DOX@EM@FA NPs with laser irradiation resulted in more than $85 \%$ cell death, which is higher than that of free DOX treatment with or without laser irradiation (with cell viability of 50\%) or sole SCPB NPs under laser irradiation (with cell viability of $80 \%$ ). These results demonstrate that the combinational photothermal/chemotherapy using SCPB@DOX@EM@FA NPs produced a significant synergistic anti-tumor efficacy. Moreover, FACS assay further verified the synergistic anti-tumor efficacy caused by dual therapy. As shown in Fig. 5C, the ratio of apoptosis to necrotic cells after with synergistic chemotherapy/PTT was $93.6 \%$, which was much higher than that of SCPB@DOX@EM@FA NPs (38.5\%) treatment or SCPB@DOX NPs treatment with laser (37\%). In addition, the rate of apoptosis and necrotic cells increased $12.5 \%$ after with SCPB@DOX@EM@FA NPs treatment relative to SCPB@DOX@EM NPs. These results demonstrate that the tumors cells damaged more significantly due to the targeting ability and synergistic chemotherapy/PTT. Finally, the protein levels of apoptosis-related genes were detected. As shown in Fig. 5D, the level of apoptosis-related proteins of NF-KB, IKK, PARP, and Bcl-2 was down-regulated compared with the control group. In contrary, the up-regulated expression of apoptotic protein BAX, cytochrome $\mathrm{C}$ and caspase-3 were found in the tumor cells treated with DOX, indicating a considerably enhanced apoptosis after with combinational treatment (SCPB@DOX@EM@FA NPs + laser irradiation) (Fig. 5E and S4†).

\section{Long circulation and tumor targeting of SCPB@DOX@EM@FA NPS}

Photoacoustic imaging (PA) was performed to visualize the distribution of SCPB@DOX@EM@FA NPs. Fig.6A indicated that the PA signal intensity significantly enhanced with the 
A

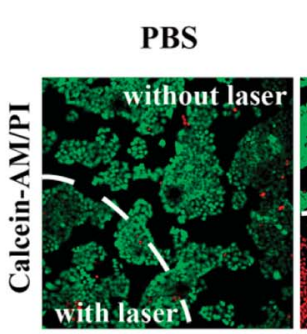

B

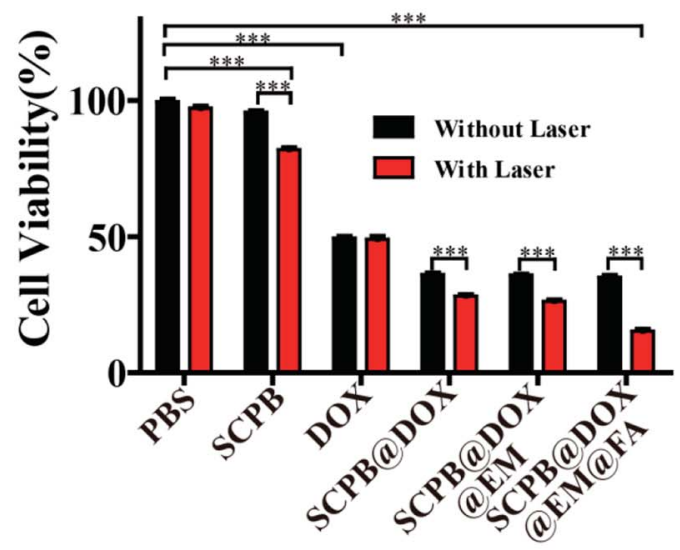

D

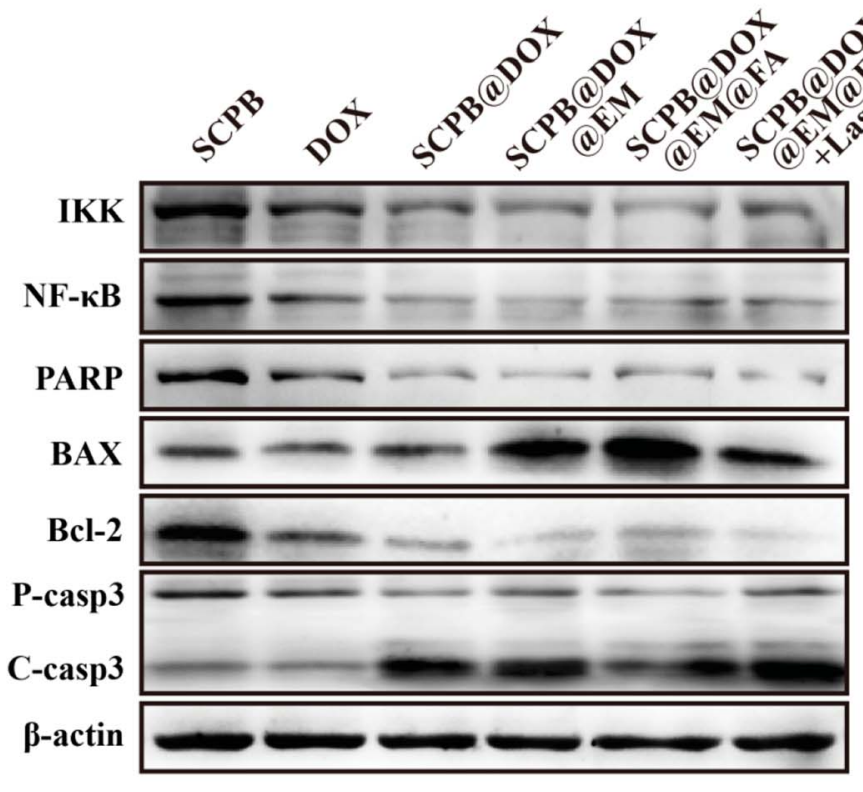

C

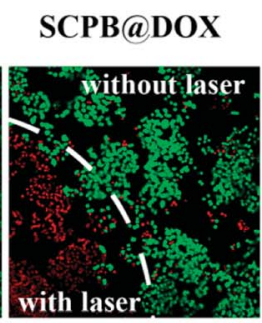

SCPB@DOX aEM@FA
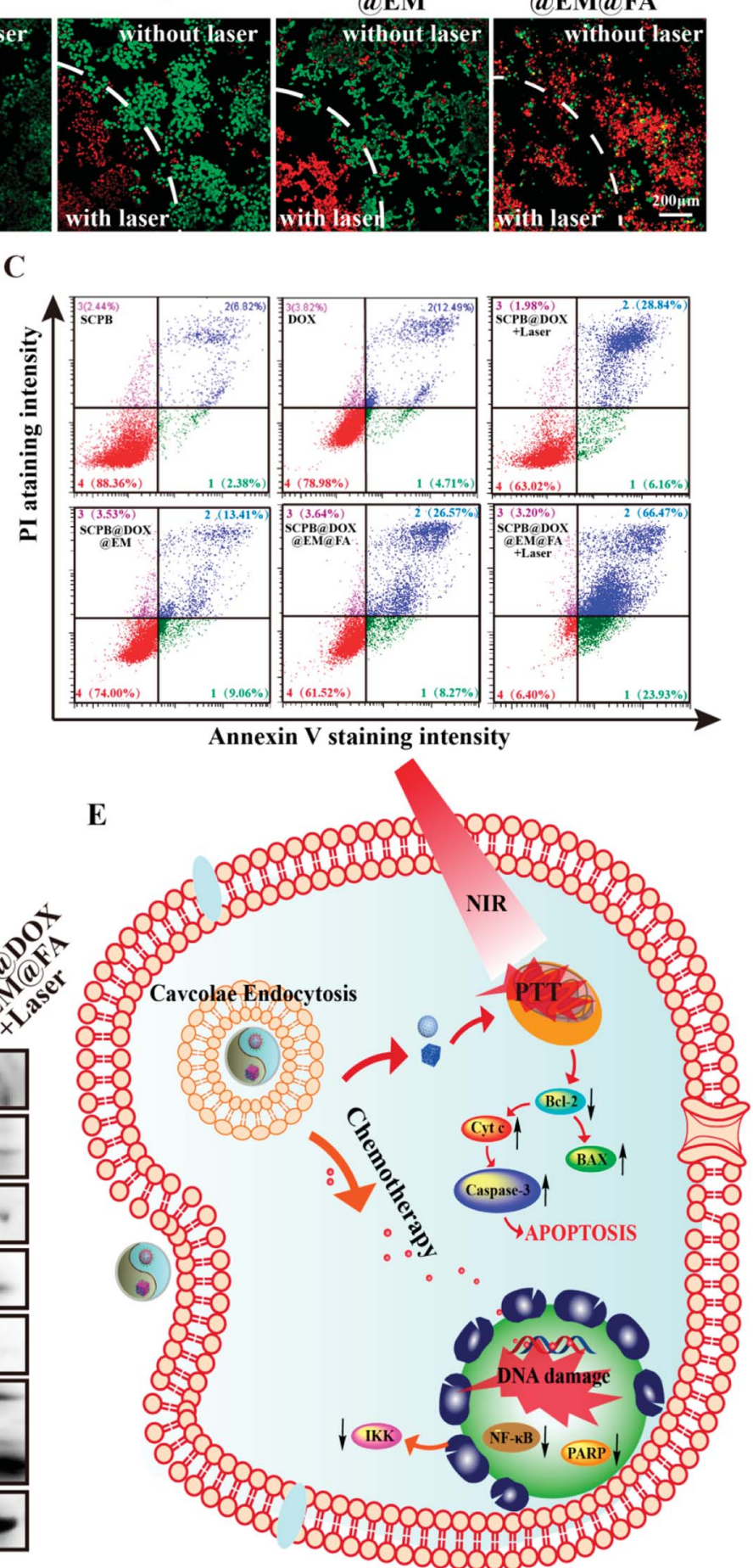

Fig. 5 In vitro anti-tumor activity of SCPB@DOX@EM@FA NPs. (A) Live/dead cell staining (scale bar = $200 \mu \mathrm{m})$. (B) MTT assay. (C) Flow cytometry analysis of HeLa cells after treatment with PBS, SCPB NPs, DOX, SCPB@DOX NPs, SCPB@DOX@EM NPs and SCPB@DOX@EM@FA NPs subjected to $808 \mathrm{~nm}$ laser irradiation. The dashed curves denote the boundary of laser irradiation spots. (D) Protein level detection of IKK, NF- $\kappa B$, caspase3, PARP, BAX, and Bcl-2 after different treatment. (E) Illustration of the SCPB@DOX@EM@FA NPs induced apoptosis pathway. Data are shown as mean standard deviation (SD), $n=3$. ( $P$ value: $* * * P<0.001)$.

concentration increase of SCPB NPs, which showed a significant concentration-dependent manner. Encouraged by the in vitro finding, PA imaging in vivo was assessed using the cervical tumor xenograft model. As shown in Fig. 6B, the PA signal at the tumor site is rather weak before injection of SCPB@DOX@EM@FA NPs. After $36 \mathrm{~h}$ post-injection, the PA signal increased 
approximately 3-fold compared with the background signal (Fig. 6C). These findings clearly indicate that the SCPB@DOX@EM@FA NPs could be hopefully applied as an acoustics agent for tumor diagnosis.

Although the aim of multi-modal cancer therapy is to obtain an effective accumulation of NPs within the tumor region, the strong clearance of macrophages to general nano-drug extremely reduced the drug concentration of circulation in vivo, which accordingly affected the therapy efficiency. Owing to the presence of macrophage recognition signal of CD47, the EM membrane camouflaged PB NPs can significantly extend circulation time in vivo by effectively avoiding the phagocytosis. ${ }^{43}$ By monitoring the blood retention of different NPs, approximately $23.21 \%$ dose per gram of blood ( $\left.\% \mathrm{ID}^{-1}\right)$ of SCPB@EM@FA NPs in the blood circulation after intravenous injection for $48 \mathrm{~h}$ were obtained, which was remarkably higher than that of naked SCPB NPs $\left(7.08 \% \mathrm{ID} \mathrm{g}^{-1}\right)$. Meanwhile, halftime for SCPB@EM@FA NPs in vivo increased 2.5-fold compared with that of naked SCPB NPs ( $8.6 \mathrm{~h}$ vs. $3.5 \mathrm{~h}$ ) (Fig. 6D). These data demonstrate that EM camouflage helps SCPB NPs stealthy to the host immune system. ${ }^{44}$ ICP-OES analysis also indicated the lower distribution of SCPB@DOX@EM@FA NPs
A

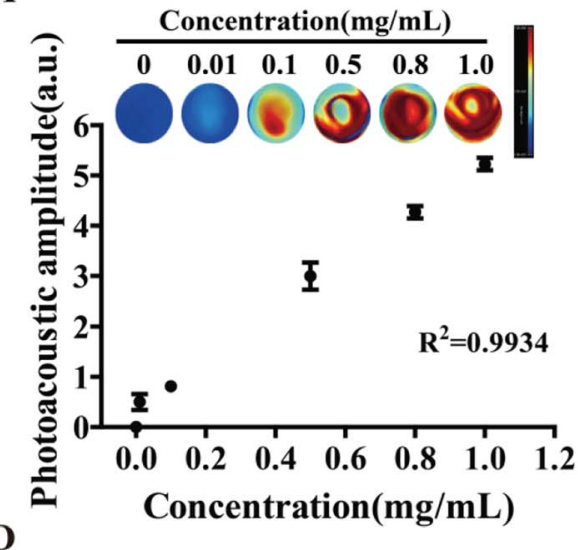

D
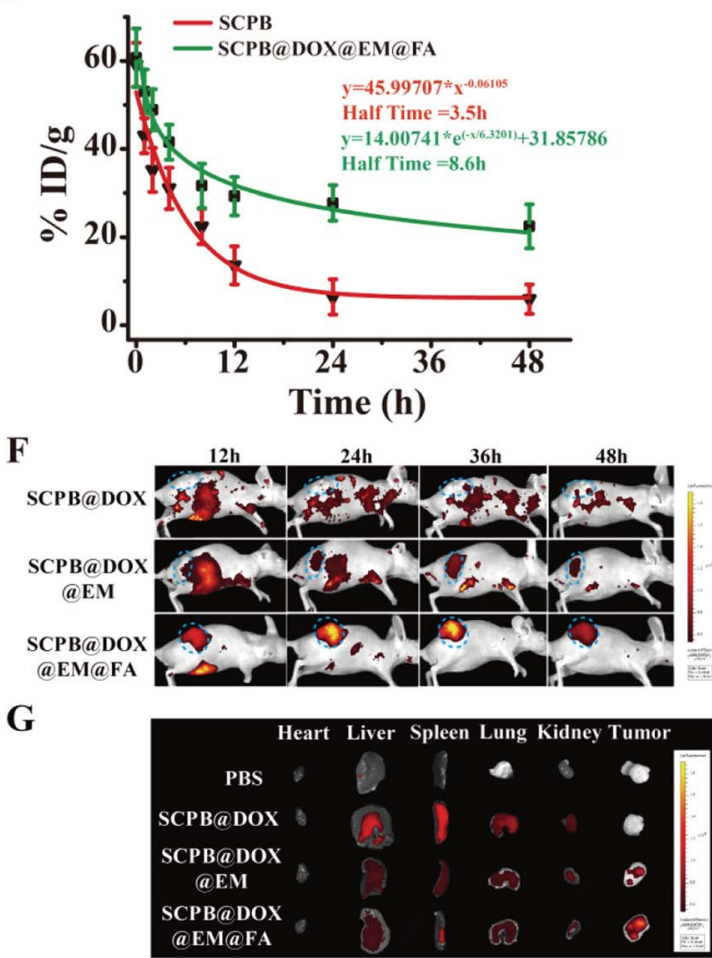

B

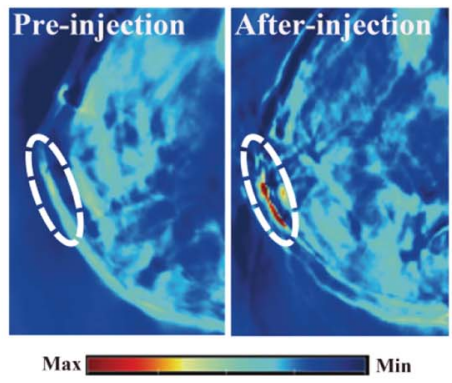

C

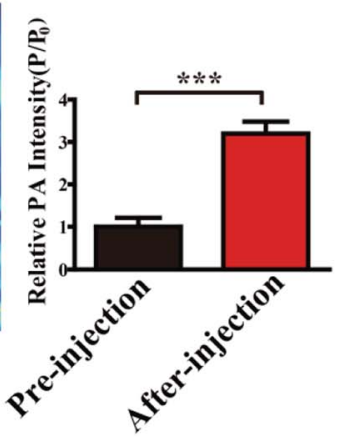

E

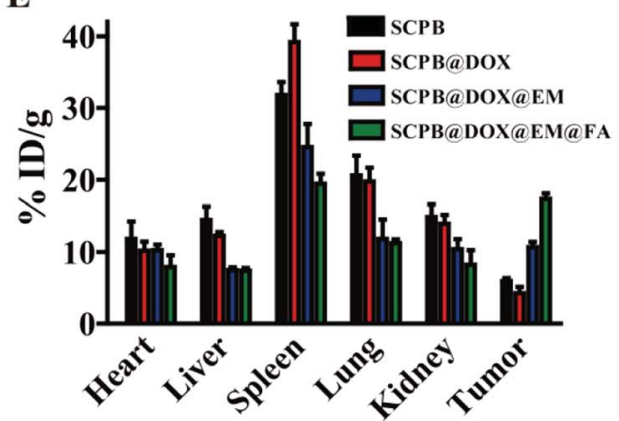

H

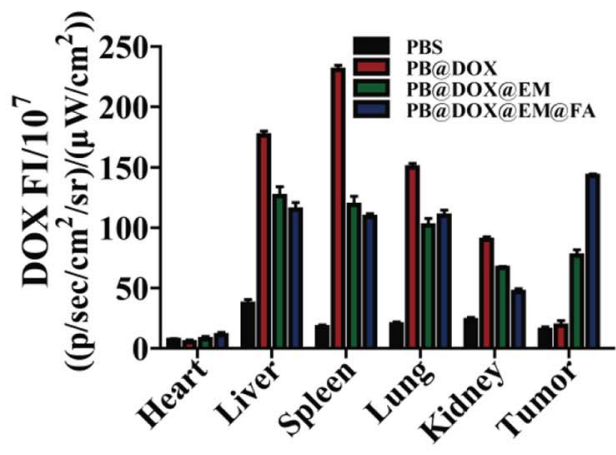

Fig. 6 (A) PA mapping of SCPBADOX@EMaFA NPs at different concentrations. PA imaging (B) and the quantification analysis (C) of the HeLatumor-bearing mice pre-injection or post-injection of SCPB@DOX@EM@FA NPs. (D) In vivo pharmacokinetic curves over a span of $48 \mathrm{~h}$ after intravenous injection of SCPB NPs or SCPBCDOX@EM@FA NPs. (E) Bio distribution of SCPB NPs, SCPB@DOX NPs, SCPB@DOX@EM NPs or SCPB@DOX@EM@FA NPs after injection for $48 \mathrm{~h}$. In vivo fluorescence images (F) of cervical tumor-bearing nude mice at 12, 24, 36 and $48 \mathrm{~h}$ after tail vein injection of SCPB@DOX NPs, SCPB@DOX@EM NPs and SCPB@DOX@EM@FA NPs; ex vivo fluorescence images (G) and quantification analysis $(\mathrm{H})$ of main organs from cervical tumor-bearing nude mice after $48 \mathrm{~h}$ tail vein injection of PBS, SCPB@DOX NPs, SCPB@DOX@EM NPs, and SCPBCDOX@EMaFA NPs. Data are shown as mean standard deviation (SD), $n=6$. ( $P$ value: $* * * P<0.001)$. 
in the heart, liver, spleen, lung, and kidney comparing with bare SCPB NPs, which suggested the capability of EM to reduce the uptake of the reticuloendothelial system of these organs. In the contrary, a higher amount of SCPB@DOX@EM@FA NPs accumulation was observed in the tumor compared with that of SCPB@DOX NPs and other groups (Fig. 6E). These results indicate that the EM coating and FA modified nanomaterials enhanced the accumulation in the tumor, which can accordingly reduce the side effects of chemotherapy drugs to normal tissues.

To more precisely monitor the tumor-targeting ability and distribution of nanomaterials, whole-body live imaging was performed at the course of $48 \mathrm{~h}$. Fig. $6 \mathrm{~F}$ showed that the fluorescence signal in three treatment group was mainly observed at $12 \mathrm{~h}$ post-injection. However, compared to SCBP@DOX NPs treatment, fluorescence signals are concentrated at the tumor site by SCPB@DOX@EM@FA NPs rather than being randomly distributed in various tissues. In addition, by observing the fluorescence signal distribution in the tumor-bearing mice, it was found that the strong fluorescence signal appeared in the tumor after administration with SCPB@DOX@EM@FA NPs for $48 \mathrm{~h}$ (Fig. 6G). It can be clearly seen from the quantitative analysis of fluorescence in Fig. $6 \mathrm{H}$, the intensity of the fluorescence signal in the tumor region of SCPB@DOX@EM@FA NPs group was at least 2.5-fold higher than that of SCPB@DOX@EM NPs group. This result demonstrates that the specific interaction between FA and FA receptor of HeLa cells accelerated the accumulation of SCPB@DOX@EM@FA NPs at the tumor region.

\section{Tumor growth inhibition mediated by SCPB@DOX@EM@FA NPs in vivo}

In vivo thermal imaging was investigated to ensure that the desired temperature change was achieved at the tumor site of the mice $24 \mathrm{~h}$ after intravenous injection of SCPB@DOX@EM@FA NPs. Fig. 7B showed no significant temperature increase of tumor site was observed in PBS treatment group after with $5 \mathrm{~min}$ of laser irradiation, while the temperature of the tumor site with post-injection of SCPB@DOX NPs and SCPB@DOX@EM@FA NPs increased $10.0{ }^{\circ} \mathrm{C}$ and $26.0{ }^{\circ} \mathrm{C}$, respectively under same conditions. These results clearly indicated that only the existence of PB NPs could directly cause temperature increase at tumor region. Meanwhile, FA modification on the PB NPs increased the local concentration of SCPB@DOX@EM@FA NPs comparing with SCPB at the tumor site, which provides the possibility for efficient thermal therapy. By comparing with the previous study, ${ }^{\mathbf{5} 10}$ compared with $808 \mathrm{~nm}$ laser irradiation group, the SCPB@DOX@EM@FA NPs $\left(23.0{ }^{\circ} \mathrm{C}\right)$ caused temperature change is higher than PEG and folic acid co-modified $\left(15.9{ }^{\circ} \mathrm{C}\right)$ or simple erythrocyte membrane-encapsulated PB NPs $\left(19.5{ }^{\circ} \mathrm{C}\right)$. The reason for this advantage is not only because of the targeted camouflage effect of folic acid modification and film encapsulation but also due to the excellent photothermal properties brought by the mixed shape PB NPs.
Then, the in vivo synergistic photothermal-/chemotherapy of SCPB@DOX@EM@FA NPs were studied using cervical tumor-bearing BALB/c mice model (Fig. 7A). As shown in Fig. 7C, no significant difference in body weight among all groups were observed over a span of 18 days after repeated injection of NPs, indicating that these NPs (SCPB@DOX) camouflaged with EM membrane have no overall side effects on the mice. In addition, the tumor growth inhibition rate (TGI) of the laser irradiation group and the DOX group was $3.5 \%$ and $53.1 \%$, respectively. However, the combination of laser irradiation with SCPB@DOX@EM NPs showed a significant TGI rate of $88.2 \%$. Despite having such a significant therapeutic effect, the tumor still has a certain risk of recurrence. Fortunately, when SCPB@DOX@EM@FA NPs was combined with laser irradiation, the TGI rate increased to 99.6\% which provided a possibility to completely eradicate the tumor (Fig. 7D). The digital photograph in Fig. 7E also shows that the tumor size after synergistic treatment is smaller than the other groups. Furthermore, the result of H\&E and TUNEL-staining demonstrated that the structure of tumor tissue was broken and a large number of tumor cells in the tissue were apoptotic after treatment with SCPB@DOX@EM@FA NPs and laser irradiation, compared with other groups (Fig. 7E). The strong anti-tumor ability of SCPB@DOX@EM@FA NPs with the help of laser irradiation indicated an effective accumulation of PB NPs and controllable release of DOX at tumor site due to the long blood circulation time, EPR effect and receptor-mediated endocytosis of tumor cells.

\section{Toxicity of SCPB@DOX@EM@FA NPs in vivo}

As blood-type mismatch occasionally leads to transfusion reaction and even death. ${ }^{44}$ The potential toxicity of this kind of EM-camouflaged NPs should be carefully considered in vivo. ${ }^{45}$ In this study, the blood of the same mice strain was used for preparing EM, as recommended previously. ${ }^{46}$ By systematically investigating the toxicity of this kind of NPs, it was found that no obvious hepatic toxicity was caused by SCPB\&DOX@EM@FA NPs, as reflected from normal values of liver function markers, including alanine transaminase (ALT), alkaline phosphatase (ALP) and aspartate aminotransferase (AST) (Fig. S5A $\dagger$ ). However, the level of creatinine (CREA) in the blood of DOX and SCPB@DOX NPs treatment groups increased, accompanied by the decrease of white blood cells (WBC) and granulocytes (GRAN) (Fig. S5B $\dagger$ and Table 1), indicating the renal impairment and bone marrow suppression was caused by the DOX and SCPB@DOX NPs. However, the camouflage of the erythrocyte membrane on the PB NPs significantly reduced the toxicity of DOX to the kidney, which was reflected by these normal bioparameters of other groups. In addition, no noticeable signal of organ damage was observed in the H\&E stained sections (Fig. S5C $\dagger$ ), which further indicated the biological safety of this nano-system. As fluctuation in the body weight is a direct indicator for the toxicity evaluation of nanomaterials in vivo, the bodyweight of mice with different treatment was measured. As we expected that no significant change was observed for these 
A

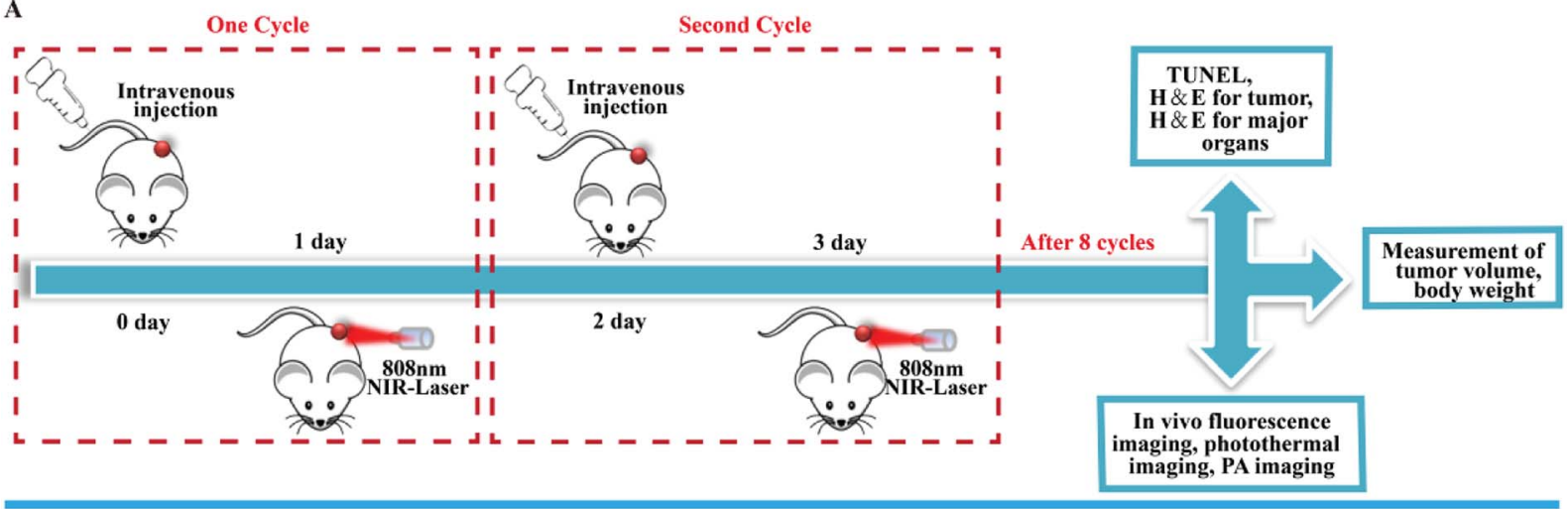

B

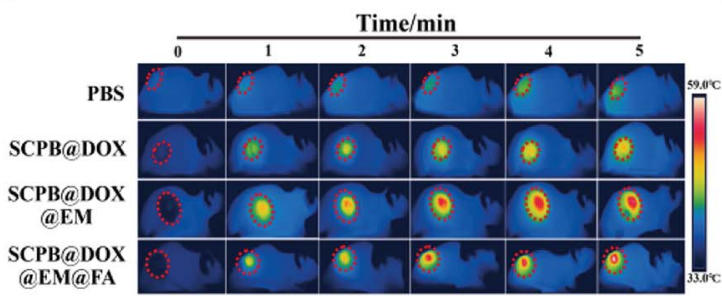

C

$\mathbf{E}$

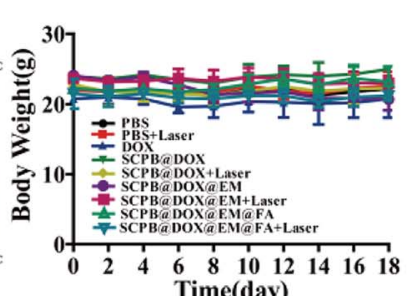

Time(day)

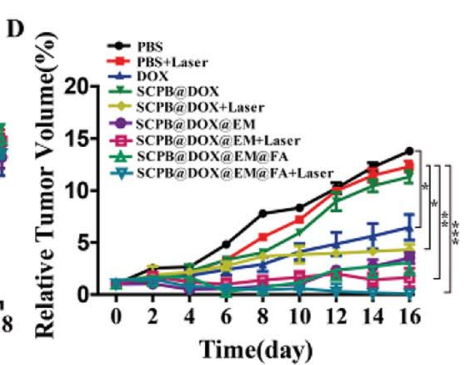

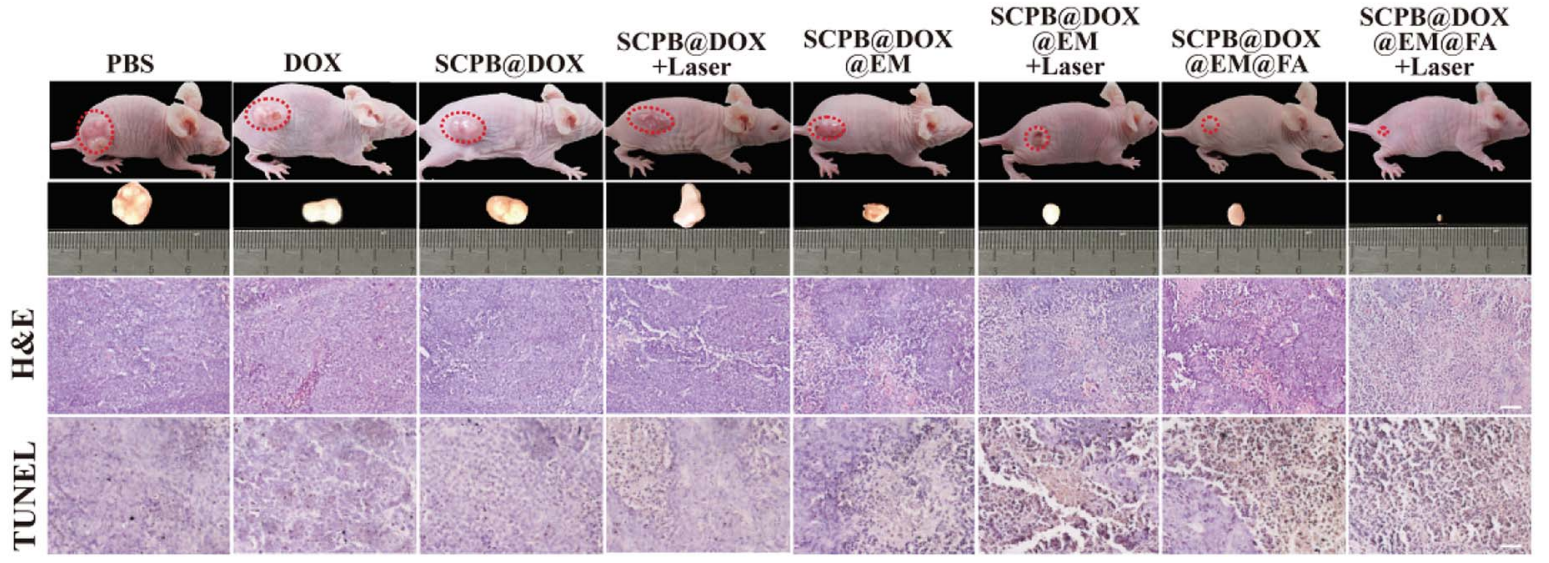

Fig. 7 Synergetic therapy of HeLa tumor-bearing mice with SCPB@DOX@EM@FA NPs. (A) Time schedule of treatments and experiments. (B) Infrared thermography of HeLa-bearing mice that were tail vein injected with PBS, SCPB@DOX NPs, SCPB@DOX@EM NPs, and SCPB@DOX@EM@FA NPs. (C) Relative body weight and (D) relative tumor volume of cervical tumor-bearing mice with different treatment. (E) Representative photographs of cervical tumor-bearing mice and tumors for each tested group (with NIR laser: $808 \mathrm{~nm}, 0.8 \mathrm{~W} \mathrm{~cm}^{-2}$ ). $\mathrm{H \& E}$ staining and TUNEL assay of the tumor tissues after different treatment (scale bar $=100 \mu \mathrm{m}$ ). $* P<0.1, * * P<0.01, * * * P<0.001$. All data represent means $\pm \mathrm{SD}(n=3)$.

Table 1 Hematological parameters of the blood collected from mice treated with PBS, DOX, SCPB@DOX, SCPB@DOX@EM, or SCPBADOXaEMaFA

\begin{tabular}{|c|c|c|c|c|c|c|}
\hline Group & $\operatorname{RBC}\left[10^{12} \mathrm{~L}^{-1}\right]$ & $\mathrm{WBC}\left[10^{9} \mathrm{~L}^{-1}\right]$ & $\operatorname{PLT}\left[10^{9} \mathrm{~L}^{-1}\right]$ & $\operatorname{GRAN}\left[10^{9} \mathrm{~L}^{-1}\right]$ & RDW [\%] & $\mathrm{MCV}[\mathrm{fL}]$ \\
\hline PBS & $9.51 \pm 0.3$ & $6.70 \pm 0.4$ & $914 \pm 176$ & $1.70 \pm 0.2$ & $16.8 \pm 0.4$ & $45.9 \pm 3.3$ \\
\hline DOX & $6.13 \pm 0.29$ & $3.40 \pm 0.3$ & $717 \pm 183$ & $1.40 \pm 0.3$ & $15.1 \pm 0.3$ & $42.6 \pm 2.2$ \\
\hline SCPB@DOX@EM & $8.70 \pm 0.81$ & $6.50 \pm 0.2$ & $907 \pm 114$ & $1.60 \pm 0.3$ & $16.3 \pm 0.5$ & $44.9 \pm 1.7$ \\
\hline SCPB@DOX@EM@FA & $8.88 \pm 0.32$ & $5.80 \pm 0.2$ & $831 \pm 136$ & $1.50 \pm 0.2$ & $15.7 \pm 0.3$ & $44.3 \pm 2.6$ \\
\hline
\end{tabular}

investigated groups. Although the above findings indicate the good biocompatibility and bio-safety of EM-coated PB, considering the difference between mice and human, the potential short- and long-term toxicity of these materials on different models should be deeply explored before using it for clinic therapy. 


\section{Conclusions}

Herein, we have demonstrated the strategy of combining photothermal effect enhanced HMPB NPs with EM camouflage and FA modification as a precise, controllable nano-drug delivery system, resulting in a controllable release and sustained accumulation of DOX. Moreover, with enhanced photothermal therapy capability of PB NPs mixture, the prepared delivery system also showed the high drug loading capacity due to the high surface area and the huge pore volumes. With the help of laser irradiation, this drug carrier was used to realize fast and precisely controllable release of the chemical drug, which is highly desirable and beneficial for the patients under the different stage of cancer. According to our point, the significant loading and desirable release performance of PB-based drug delivery system makes it an ideal alternative for loading liposoluble chemical drugs. Given the ultrahigh bio-safety, low price and high combinational therapy efficiency, the proposed strategy will produce great significance for personalized therapy of various tumors by combining a membrane system with other drugs.

\section{Conflicts of interest}

The authors declare that they have no conflict of interest.

\section{Acknowledgements}

This work was partially supported by the Natural Science Foundation of China (81673579 and 81874369), Hunan Province Universities 2011 Collaborative Innovation Center of Protection and Utilization of Hu-Xiang Chinese Medicine Resources and the National Standardization Project of Traditional Chinese Medicine (Grant ZYBZH-Y- HUN-23).

\section{References}

1 J. Conde, N. Oliva, Y. Zhang and N. Artzi, Local triplecombination therapy results in tumour regression and prevents recurrence in a colon cancer model, Nat. Mater., 2016, 15, 1128.

2 Q. Feng, M.-Z. Yu, J.-C. Wang, W.-J. Hou, L.-Y. Gao, X.-F. Ma, et al., Synergistic inhibition of breast cancer by co-delivery of VEGF SiRNA and paclitaxel via vapreotide-modified coreshell nanoparticles, Biomaterials, 2014, 35, 5028-5038.

3 F. Greco and M. J. Vicent, Combination therapy: opportunities and challenges for polymer-drug conjugates as anticancer nanomedicines, Adv. Drug Delivery Rev., 2009, 61, 1203-1213.

4 H. J. Broxterman and N. H. Georgopapadakou, Anticancer therapeutics:"Addictive" targets, multi-targeted drugs, new drug combinations, Drug Resist. Updates, 2005, 8, 183-197.

5 W. Chen, K. Zeng, H. Liu, J. Ouyang, L. Wang, Y. Liu, et al., Cell Membrane Camouflaged Hollow Prussian Blue Nanoparticles for Synergistic Photothermal-/Chemotherapy of Cancer, Adv. Funct. Mater., 2017, 27, 1605795.
6 X. Deng, K. Li, X. Cai, B. Liu, Y. Wei, K. Deng, et al., A Hollow-Structured CuS@ Cu2S@ Au Nanohybrid: Synergistically Enhanced Photothermal Efficiency and Photoswitchable Targeting Effect for Cancer Theranostics, Adv. Mater., 2017, 29, 1701266.

7 Z. Sheng, D. Hu, M. Zheng, P. Zhao, H. Liu, D. Gao, et al., Smart human serum albumin-indocyanine green nanoparticles generated by programmed assembly for dualmodal imaging-guided cancer synergistic phototherapy, ACS Nano, 2014, 8, 12310-12322.

8 J. P. Thawani, A. Amirshaghaghi, L. Yan, J. M. Stein, J. Liu and A. Tsourkas, Photoacoustic-Guided Surgery with Indocyanine Green-Coated Superparamagnetic Iron Oxide Nanoparticle Clusters, Small, 2017, 13, 1701300.

9 V. Saxena, M. Sadoqi and J. Shao, Degradation kinetics of indocyanine green in aqueous solution, J. Pharm. Sci., 2003, 92, 2090-2097.

10 Y. Yang, L. Jing, X. Li, L. Lin, X. Yue and Z. Dai, Hyaluronic acid conjugated magnetic Prussian blue@ quantum dot nanoparticles for cancer theranostics, Theranostics, 2017, 7, 466-481.

11 K. Yang, L. Hu, X. Ma, S. Ye, L. Cheng, X. Shi, et al., Multimodal imaging guided photothermal therapy using functionalized graphene nanosheets anchored with magnetic nanoparticles, Adv. Mater., 2012, 24, 1868-1872.

12 H. Ke, J. Wang, Z. Dai, Y. Jin, E. Qu, Z. Xing, et al., Gold-nanoshelled microcapsules: a theranostic agent for ultrasound contrast imaging and photothermal therapy, Angew. Chem., Int. Ed., 2011, 50, 3017-3021.

13 S. M. Lee, H. Park and K. H. Yoo, Synergistic cancer therapeutic effects of locally delivered drug and heat using multifunctional nanoparticles, Adv. Mater., 2010, 22, 40494053.

14 J. Yang, J. Choi, D. Bang, E. Kim, E. K. Lim, H. Park, et al., Convertible organic nanoparticles for near-infrared photothermal ablation of cancer cells, Angew. Chem., Int. Ed., 2011, 50, 441-444.

15 C. Tong, X. Zhang, J. Fan, B. Li, B. Liu, M. Daniyal, et al., PEGylated mBPEI-rGO nanocomposites facilitate hepotocarcinoma treatment combining photothermal therapy and chemotherapy, Sci. Bull., 2018, 63, 935-946.

16 X. Liang, Z. Deng, L. Jing, X. Li, Z. Dai, C. Li, et al., Prussian blue nanoparticles operate as a contrast agent for enhanced photoacoustic imaging, Chem. Commun., 2013, 49, 1102911031.

17 L. Cheng, H. Gong, W. Zhu, J. Liu, X. Wang, G. Liu, et al., PEGylated Prussian blue nanocubes as a theranostic agent for simultaneous cancer imaging and photothermal therapy, Biomaterials, 2014, 35, 9844-9852.

18 X. Cai, W. Gao, M. Ma, M. Wu, L. Zhang, Y. Zheng, et al., A Prussian Blue-Based Core-Shell Hollow-Structured Mesoporous Nanoparticle as a Smart Theranostic Agent with Ultrahigh pH-Responsive Longitudinal Relaxivity, Adv. Mater., 2015, 27, 6382-6389.

19 X. Peng, R. Wang, T. Wang, W. Yang, H. Wang, W. Gu, et al., Carbon dots/Prussian blue satellite/core nanocomposites for 
optical imaging and photothermal therapy, ACS Appl. Mater. Interfaces, 2017, 10, 1084-1092.

20 T. Kim, J. E. Lemaster, F. Chen, J. Li and J. V. Jokerst, Photoacoustic Imaging of Human Mesenchymal Stem Cells Labeled with Prussian Blue-Poly (l-lysine) Nanocomplexes, ACS Nano, 2017, 11, 9022-9032.

21 B. T. Luk, R. H. Fang, C.-M. J. Hu, J. A. Copp, S. Thamphiwatana, D. Dehaini, et al., Safe and immunocompatible nanocarriers cloaked in RBC membranes for drug delivery to treat solid tumors, Theranostics, 2016, 6, 1004.

22 C.-M. J. Hu, R. H. Fang, B. T. Luk and L. Zhang, Nanoparticle-detained toxins for safe and effective vaccination, Nat. Nanotechnol., 2013, 8, 933.

23 F. Pi, D. W. Binzel, T. J. Lee, Z. Li, M. Sun and P. Rychahou, Nanoparticle orientation to control RNA loading and ligand display on extracellular vesicles for cancer regression, Nat. Nanotechnol., 2018, 13, 82.

24 C.-M. J. Hu, R. H. Fang, J. Copp, B. T. Luk and L. Zhang, A biomimetic nanosponge that absorbs pore-forming toxins, Nat. Nanotechnol., 2013, 8, 336.

25 L. Zhang, D. Wang, K. Yang, D. Sheng, B. Tan, Z. Wang, et al., Mitochondria-Targeted Artificial "Nano-RBCs" for Amplified Synergistic Cancer Phototherapy by a Single NIR Irradiation, Adv. Sci., 2018, 5, 1800049.

26 D. Wang, H. Dong, M. Li, Y. Cao, F. Yang, K. Zhang, et al., Erythrocyte-Cancer Hybrid Membrane Camouflaged Hollow Copper Sulfide Nanoparticles for Prolonged Circulation Life and Homotypic-Targeting Photothermal/ Chemotherapy of Melanoma, ACS Nano, 2018, 6, 5241-5252.

27 F. Pierigè, S. Serafini, L. Rossi and M. Magnani, Cell-based drug delivery, Adv. Drug Delivery Rev., 2008, 60, 286-295.

28 V. R. Muzykantov, Drug delivery by red blood cells: vascular carriers designed by mother nature, Expert Opin. Drug Delivery, 2010, 7, 403-427.

29 P. S. Low, W. A. Henne and D. D. Doorneweerd, Discovery and development of folic-acid-based receptor targeting for imaging and therapy of cancer and inflammatory diseases, Acc. Chem. Res., 2007, 41, 120-129.

30 S. D. Weitman, R. H. Lark, L. R. Coney, D. W. Fort, V. Frasca, V. R. Zurawski, et al., Distribution of the folate receptor GP38 in normal and malignant cell lines and tissues, Cancer Res., 1992, 52, 3396-3401.

31 K. Tian, X. Jia, X. Zhao and P. Liu, pH/Reductant DualResponsive Core-Cross-Linked Micelles via Facile in situ ATRP for Tumor-Targeted Delivery of Anticancer Drug with Enhanced Anticancer Efficiency, Mol. Pharm., 2016, 13, 2683-2690.

32 S. Santra, C. Kaittanis, O. J. Santiesteban and J. M. Perez, Cell-specific, activatable, and theranostic prodrug for dualtargeted cancer imaging and therapy, J. Am. Chem. Soc., 2011, 133, 16680-16688.

33 Y. Zhao, H. Pan, Y. Lou, X. Qiu, J. Zhu and C. Burda, Plasmonic $\mathrm{Cu} 2-$ x S nanocrystals: optical and structural properties of copper-deficient copper (I) sulfides, J. Am. Chem. Soc., 2009, 131, 4253-4261.

34 D. K. Roper, W. Ahn and M. Hoepfner, Microscale heat transfer transduced by surface plasmon resonant gold nanoparticles, J. Phys. Chem. C, 2007, 111, 3636-3641.

$35 \mathrm{~W}$. Hou and S. B. Cronin, A review of surface plasmon resonance-enhanced photocatalysis, Adv. Funct. Mater., 2013, 23, 1612-1619.

36 Y. Liu, G. Shu, X. Li, H. Chen, B. Zhang, H. Pan, et al., Human HSP70 Promoter-Based Prussian Blue Nanotheranostics for Thermo-Controlled Gene Therapy and Synergistic Photothermal Ablation, Adv. Funct. Mater., 2018, 1802026.

37 Q. Tian, F. Jiang, R. Zou, Q. Liu, Z. Chen, M. Zhu, et al., Hydrophilic Cu9S5 nanocrystals: a photothermal agent with a $25.7 \%$ heat conversion efficiency for photothermal ablation of cancer cells in vivo, ACS Nano, 2011, 5, 97619771.

38 G. Fu, W. Liu, S. Feng and X. Yue, Prussian blue nanoparticles operate as a new generation of photothermal ablation agents for cancer therapy, Chem. Commun., 2012, 48, 11567-11569.

39 M. Gao, A. Hu, X. Sun, C. Wang, Z. Dong, L. Feng, et al., Photosensitizer decorated red blood cells as an ultrasensitive light-responsive drug delivery system, ACS Appl. Mater. Interfaces, 2017, 9, 5855-5863.

40 X. Cao, F. Feng, Y. Wang, X. Yang, H. Duan and Y. Chen, Folic acid-conjugated graphene oxide as a transporter of chemotherapeutic drug and siRNA for reversal of cancer drug resistance, J. Nanopart. Res., 2013, 15, 1965.

41 X. Sun, L. Wei, H. Chen and W. Liang, Advances in the study of organelles targeting nanocarriers, Acta Pharm. Sin., 2009, 44, 838-844.

42 L. Ding, C. Yao, X. Yin, C. Li, Y. Huang, M. Wu, et al., Size, Shape, and Protein Corona Determine Cellular Uptake and Removal Mechanisms of Gold Nanoparticles, Small, 2018, 14, 1801451.

43 J.-G. Piao, L. Wang, F. Gao, Y.-Z. You, Y. Xiong and L. Yang, Erythrocyte membrane is an alternative coating to polyethylene glycol for prolonging the circulation lifetime of gold nanocages for photothermal therapy, ACS Nano, 2014, 8, 10414-10425.

44 H. Jick, B. Westerholm, M. Vessey, G. Lewis, D. Slone, W. W. Inman, et al., Venous thromboembolic disease and ABO blood type: a cooperative study, Lancet, 1969, 293, 539-542.

45 A. Nel, T. Xia, L. Mädler and N. Li, Toxic potential of materials at the nanolevel, Science, 2006, 311, 622-627.

46 L.-L. Li, J.-H. Xu, G.-B. Qi, X. Zhao, F. Yu and H. Wang, Coreshell supramolecular gelatin nanoparticles for adaptive and "on-demand" antibiotic delivery, ACS Nano, 2014, 8, 49754983. 